University of Louisville

ThinkIR: The University of Louisville's Institutional Repository

Electronic Theses and Dissertations

$12-2004$

\title{
Novel synthesis techniques for nanostructures.
}

Romaneh Jalilian 1977-

University of Louisville

Follow this and additional works at: https://ir.library.louisville.edu/etd

\section{Recommended Citation}

Jalilian, Romaneh 1977-, "Novel synthesis techniques for nanostructures." (2004). Electronic Theses and Dissertations. Paper 675.

https://doi.org/10.18297/etd/675

This Master's Thesis is brought to you for free and open access by ThinkIR: The University of Louisville's Institutional Repository. It has been accepted for inclusion in Electronic Theses and Dissertations by an authorized administrator of ThinkIR: The University of Louisville's Institutional Repository. This title appears here courtesy of the author, who has retained all other copyrights. For more information, please contact thinkir@louisville.edu. 


\title{
NOVEL SYNTHESIS TECHNIQUES
}

\section{FOR NANOS'TRUCTURES}

\author{
By \\ Romaneh Jalilian \\ B.S., Shahid Beheshti University, Tehran, Iran, 2000
}

\author{
A Thesis \\ Submitted to the Faculty of the \\ Graduate School of the University of Louisville \\ in Partial Fulfillment of the Requirements \\ for the Degree of
}

Master of Science

Department of Physics University of Louisville Louisville, Kentucky

December 2004 


\title{
NOVEL SYNTHESIS THECHNIQUES
}

FOR NANOSTRUCTURES

\author{
By \\ Romaneh Jalilian \\ B.S., Shahid Beheshti University, Tehran, Iran, 2000 \\ A Thesis approved on \\ December 2004 \\ by The following Thesis Committee: \\ Dr. Gamini Sumanasekera, Thesis Director \\ Dr. Shi Yu Wu \\ Dr. Robert W. Cohn
}




\section{ACKNOWLEDGEMENTS}

I would like to thank my thesis advisor, Dr. Gamini Sumanasekera, for continual guidance and encouragement throughout the research. I would like to thank him for giving me an opportunity to put myself in this new developing field, which is at the forefront of science. I am thankful to my husband, Mehdi Yazdanpanah, for being a continuous source of love, motivation, support and technical advice. I would also like to thank Dr. Shi-Yu Wu and Dr. Robert W. Cohn for giving their valuable time to examine this thesis. I would like to thank Dr. Steven A. Harfenist, Dr. Martin Williams, Dr. Bruce. W. Alphanar, Dr. B. K. Pradhan, Dr. Gugang Chen, for their scientific advice and help. I am grateful of Prashant Gopinath and Aditya Mohite for sharing their knowledge. I am thankful to Mr. Keith Gowen for his machine shop help. I am thankfull to Mr. Charls Coven for the help of electronic shop . I am also thankful to my colleagues Sharvil and Jose for making the lab an appropriate place to work. 


\section{ABSTRACT \\ NOVEL SYNTHESIS THECNIQURE FOR NANOSTRUCTURES}

Romaneh Jalilian

December, 2004

Crystalline $\beta-\mathrm{Ga}_{2} \mathrm{O}_{3}$ nanowires with two distinct morphologies have been synthesized through simple physical evaporation of Te doped GaAs powder in argon atmosphere. The structure of the nanowires was characterized by SEM, TEM, XRD, EDX, and Raman spectroscopy. Nanowires as long as hundreds of micrometers with diameters in the range of 20-200 nm have been produced with a high yield. Absence of Tellurium in the nanowires indicates that the growth mechanism is not VLS based. The role of Tellurium in the growth process is not clear. Substitution of sulfur in place of tellurium resulted in similar nanostructures. One of the morphologies of the nanowires exhibits herringbone structure and the TEM images show hexagonal crystallites ordered in regular spacing along the nanowire axis. The crystal plane of the nanowire is parallel to one of the facets of the crystallite. The other morphology is essentially platelets and free of hexagonal crystallites.

Laser assisted catalyst growth process has been employed to synthesize various semiconducting nanowires, heterostructural nanowires and single walled carbon nanotubes. Structural characterization and physical properties of individual nanostructures have been explored. 


\section{TABLE OF CONTENTS}

Page

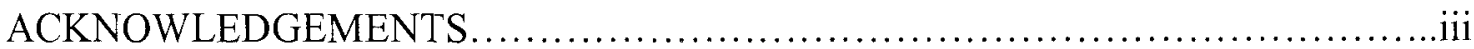

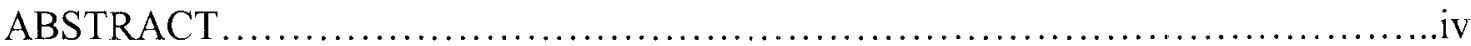

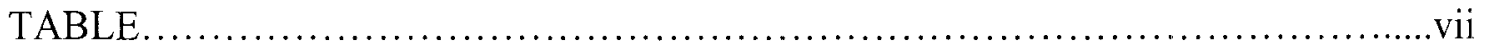

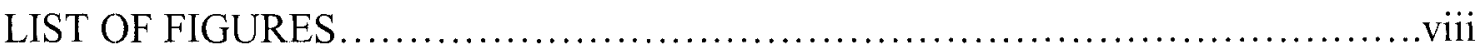

\section{CHAPTER}

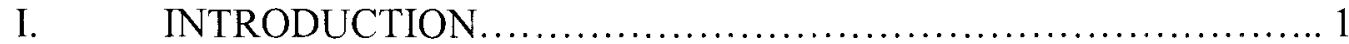

Nanoscience and Nanotechnology...............................1

Nanoparticles..............................................

Nanotubes..........................................................

Nanowires................................................. 8

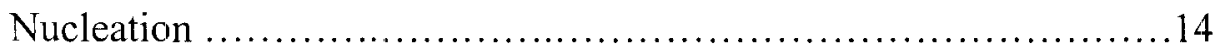

Chemical Vapor Deposition..................................... 15

Physical Vapor Deposition......................................16

Laser Ablation (Pulse Laser Vaporization) .........................17

Transmission Electron Microscopy.................................20

Scanning Electron Microscopy................................22

X-Ray Powder Diffraction...................................24

Energy Dispersive X-ray Spectroscopy.........................26

Raman Spectroscopy........................................27 
II. EXPERIMENT.

Synthesis

Design and manufacture of the chemical vapor deposition system ....30

CVD growth of Gallium oxide .32

Design and manufacture of the pulse laser vaporization system. 33

PLV growth of some nanowires .37

III. CHARACTERIZATION ...............................................42

Result and discussion.........................................42

$\mathrm{Ga}_{2} \mathrm{O}_{3}$ nanowires and herringbone structures......................42

Gallium arsenide nanowires..........................................51

Gallium phosphate nanowires...................................53

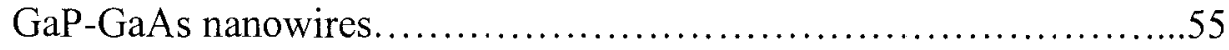

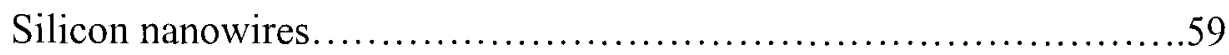

Single wall carbon nanotubes....................................60

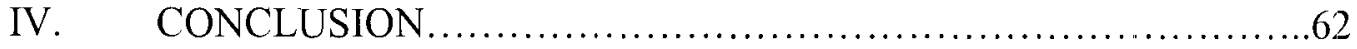

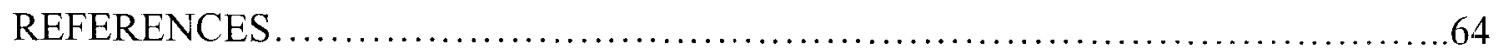

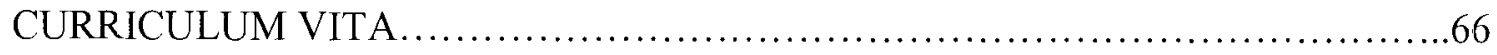




\section{LIST OF TABLES}

TABLE

1. Growth summary of some nanowires......................................11

2. Summary of $\mathrm{Ga}_{2} \mathrm{O}_{3}$ experimental results.................................43 


\section{LIST OF FIGURES}

Figure $\quad$ Page

1. Gold Nanoparticles by laser ablation in aqueous solution of surfactant ............4

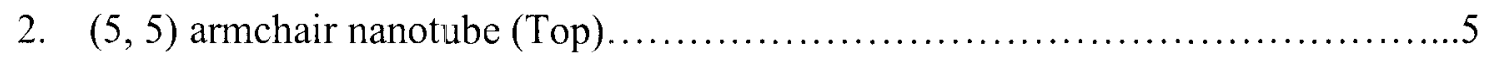

2a. $(9,0)$ zigzag nanotube (middle) and $(10,5)$ chiral nanotube. The diameter

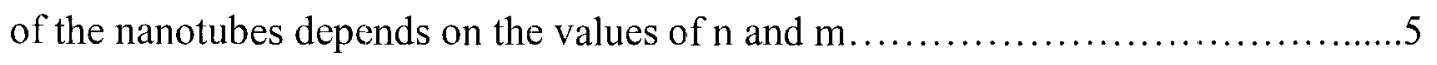

3. Schematic diagrams of the modeled, single wall carbon field effect transistor........7

4. AFM image of CNT in Carbon Nanotube Field-Effect Transistor (CNTFET),

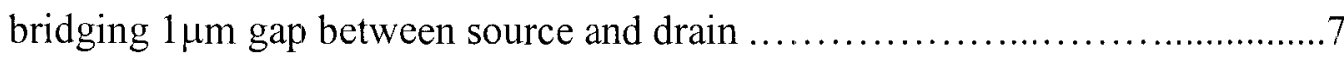

5. VLS mechanism of Si nanowire synthesis using Fe as catalyst nanoparticle ........9

6. Pseudobinary phase diagram for Au and Ga As.............................. 12

7a. Low resolution TEM image of GaAs Nanowires, the catalyst particle is attached to the tip of the NW

7b. FETEM image of the crystalline structure of GaAs nanowires growth by

VLS method

8a. GaAs/GaP nanowire TEM image and the diffraction pattern.

$8 b, c, d$ and e. EDS result for NW, the border of the chemicals is very clear and 
sharp

9. Schematic of the formation mechanism of mono-depressive spherical particles by nucleation and aggregation.

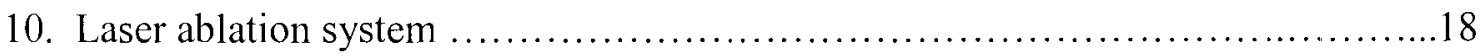

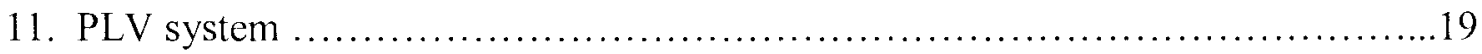

12. Laser assisted growth result of Si nanowires................................20

13. Schematic of optics and Imaging of the TEM system ............................21

14. TEM image of some dispersive Boron NWs...............................22

14a. The corresponding SAED pattern of the arrowed boron nanowires..............22

14b. HRTEM image of the arrowed boron nanowires...............................22

15. Scanning electron microscope schematic ...................................24

16. Schematic of $x$-ray emission in $\mathrm{K} \alpha$ and $\mathrm{K} \beta$ mode in an atom .......................

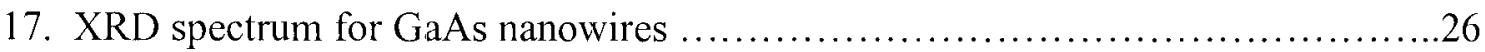

18. TEM image of the end of GaAs nanowires (the dark part at tip of wire is gold catalyst) and EDS spectrum for two white spots on the image ...................27

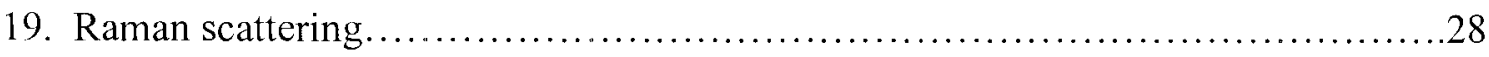

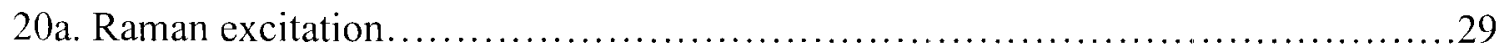

20b. Stokes and anti-stokes and Rayleigh Radiation................................29

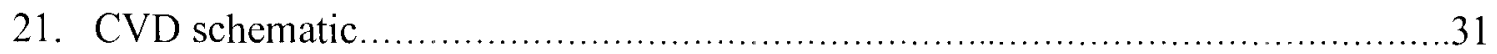

22. CVD set up in the laboratory................................................. 31

23. Schematic of Pulse laser vaporization system ................................33

24. Graph of reflectivity of mirrors vs. wavelength................................. 34 
26b. Pellet maker.

26c. Target.

27. Poco graphite target holder. .38

28. Lab view screenshot for scanning the $\mathrm{GaP} / \mathrm{GaAs}$ target 40

29. Scanning path of the laser on $\mathrm{GaP} / \mathrm{GaAs}$ target. 41

30a. $\mathrm{Ga}_{2} \mathrm{O}_{3}$ nanostructures, $\mathrm{PVD}$ growth from $\mathrm{GaAs} / \mathrm{Te}$.

30b. $\mathrm{Ga}_{2} \mathrm{O}_{3}$ nanostructure from $\mathrm{GaAs} / \mathrm{S}$ .43

30c. $\mathrm{Ga}_{2} \mathrm{O}_{3}$ nanostructure from $\mathrm{GaAs} / \mathrm{Te}$ and $\mathrm{Au}$. .43

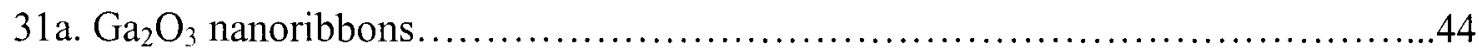

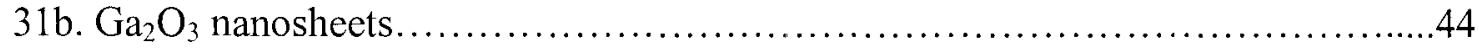

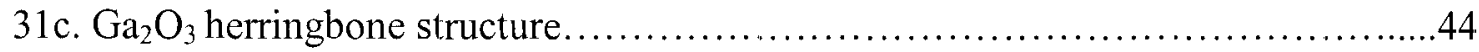

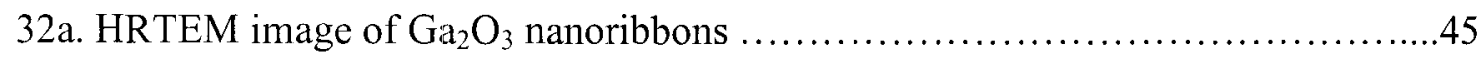

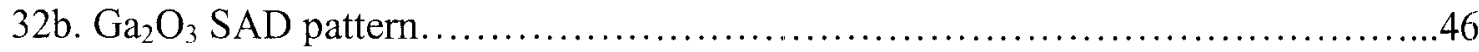

33a. TEM image of herringbone structure ....................................46

33b. SAD diffraction pattern of nanostructure ...............................46

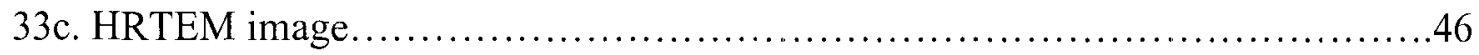

33d. $\mathrm{Ga}_{2} \mathrm{O}_{3}$ ribbons' HRETM images showing that the ribbon is not flat on the surface. .46

34. X-ray powder diffraction of the $\mathrm{Ga}_{2} \mathrm{O}_{3}$ nanostructures. 48 
35. EDS spectrum of Gallium oxide nanostructures.

36. EDS spectrum of $\mathrm{Ga}_{2} \mathrm{O}_{3}$ smaller nanostructures taken with TEM

37. Macro-Raman spectroscopy of $\mathrm{Ga}_{2} \mathrm{O}_{3}$ nanoribbons, $514.5 \mathrm{~nm}$ laser excitation....50

38. AFM image of $\mathrm{Ga}_{2} \mathrm{O}_{3}$ nanoribbons

39a. GaAs collected on silicon substrate

39b. GaAs dispersed on the silicon; straight shape. .52

39c. Dispersed curly nanowires of GaAs.

40. EDS of GaAs nanowires. 53

41a. SEM images of $\mathrm{GaP}$ nanowires deposited on a piece of silicon 54

41b. SEM images of GaP nanowires dispersed on silicon .54

42. EDS spectrum of GaP nanowires. 54

43a. The SEM image of GAP NW .55

43a, b, c. EDS mapping of Ga, P, Si substrate with nanowires sitting on the top......55

44a. SEM images of GaAs-GAP hetero-structures collected on the tube's wall. .56

44b.SEM image of dispersed $\mathrm{GaP} / \mathrm{GaAs} \mathrm{NW}$ on Si substrate with gold path .56

44c.SEM image of GaP-GaAs nanowires with Fe catalyst. .56

44d. SEM image of GaP-GaAs nanowires with Au catalyst. .56

45a. EDS data of GaP/GaAs NWs collected off the PLV tube 57

46. Macro Raman spectrum of GaAs-GaP NWs. .58 


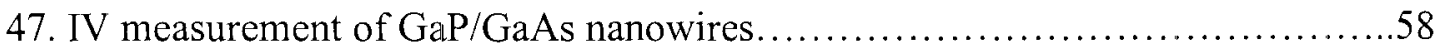

48a. SEM images of Si NWs with Au catalyst................................59

48b. SEM image of So NWs with Fe catalyst...............................59

49. SEM images of Si NWs with gold catalyst..............................60

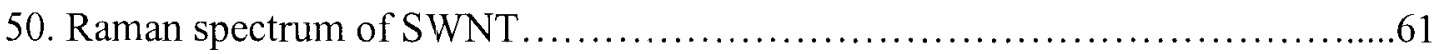




\section{CHAPTER I}

\section{INTRODUCTION}

\section{Nanoscience and Nanotechnology}

Nanophase and nanostructured materials have attracted a great deal of interest due to their potential applications in different areas such as electronics, optics, catalysis, ceramics, magnetic data storage, and nanocomposites. The unique properties of nanomaterials are determined by their size, surface structures, interparticle interaction and surface to volume ratio.

Nanomaterials are classified into 1) Nanostructured materials, such as nanowires, nanotubes, nanowebs and thin films, and 2) Nanophase materials like nanoparticles and catalysts. To distinguish nanomaterials from bulk it is important to study the unique properties of nanomaterials and their applications in science and technology.

A parameter introduced by nanomaterials is the surface/interface to volume ratio. A high percentage of surface atoms lead to many size dependent phenomena. The finite size of the particle confines the spatial distribution of electrons and causes quantized energy levels due to small size effect. This can provide deep insight into the theory of energy transitions from discrete energy levels for fundamental elements and continuum energy levels for bulk materials. This quantum confinement has applications in semiconductors, optoelectronics and non--linear optics. Another fundamental feature of nanoscience is nucleation. Nucleation and growth are two important parameters for synthesis of thin 
films and nanowires resulting in formation of crystalline particles under suitable conditions. Aggregation of these particles leads to nanocrystalline structures.

Nanomaterials have novel mechanical properties. The strength of a solid depends on its size and surface to volume ratio. In a crystalline structure strength is dependent on grain boundary sliding and energy dissipation at interfaces. By decreasing the size of the grain the hardness of the material is increased. The high surface to volume ratio determines the thermodynamic properties of the materials. A structure with less coordinated surface atoms increases the surface energy so that the diffusion of atoms happens at lower temperatures. As a result the melting temperature decreases in nanomaterials. For example, the melting temperature for bulk gold is $1063{ }^{\circ} \mathrm{C}$, but for gold nanoparticles is about $300{ }^{\circ} \mathrm{C}$. Semiconductor nano dots are zero dimensional quantum systems, in which carriers are confined and convert the density of states to a set of discrete quantum levels which is necessary for semiconductor laser function. In nanodevices quantum physics is substituted for classical physics and devices work based on tunneling of the electrons through potential barriers and a single electron can govern the function of a transistor, switch or memory element. The discovery of $\mathrm{C}_{60}$ (Fullerene) is another new field in carbon related nanomaterials. Carbon nanotubes are concentric cylinders of graphite sheets and they appear as single cylinders called single wall carbon nanotubes (SWNT), as two concentric cylinders called double wall carbon nanotubes (DWNT), or multi concentric tubes called multi wall carbon nanotubes (MWNT). The finite dimension of carbon nanotubes and the chirality angle, which determines how the graphite sheet is rolled, result in unique electronic properties; such as ballistic quantum conductance, semiconductor junctions, and electron field emission. The structure of carbon nanotubes 
leads to an extraordinary mechanical strength and high elastic strength. There are numerous applications for nanotubes related to their structure and properties. They can be added to a chain of polymer in different ways or used a fine tip for Atomic force microscopy (AFM) or scanning tunneling microscopy (STM). There are a vast number of projects in progress to apply carbon nanotubes for gas sensor or electronic devices and transistors. [1]

There are three important steps in nanotechnology; material preparation, property measurement and device fabrication. Nanomaterials such as nano particles, nanowebs, nanorods, nanowires, nanotubes and more nanostructures have been prepared and some of the nanostructures have been characterized.

\section{Nanoparticles}

Nanoparticles are currently made of a wide variety of materials. One of the new generations of materials is ceramics, which are metal oxides such as titanium, zinc, aluminum and iron oxides, as well as metal nanoparticles. Silicate nanoparticles, a kind of ceramic nanoparticle, are flakes about $1 \mathrm{~nm}$ thick and 100 to $1000 \mathrm{~nm}$ across. The nanoparticles can be incorporated into polymers during polymerization or by melt compounding. Pure metal nanoparticles can be merged into a solid by chemical reaction of a solvent and substrate; these nanoparticles create a thin layer or a film.

Mass production of nanoparticles is important in nanoelectronics, for example, insulating matrices encapsulating metallic nanoparticles exhibit nonlinear optical properties which are size dependent. Any synthesis technique for particles to be used in these applications must produce high quality, individual, nanoparticles with narrow size distributions (mono- dispersed). Spherical, high quality, nanometer sized particles can be made from a 
wide variety of materials via laser ablation of micro particles (LAM). The finite volume contained within a target microparticle significantly increases the importance of the breakdown-induced shock wave in nanoparticle formation [2]. The nanoparticles can be obtained via other methods of laser ablation for example in an aqueous solution [3].(Fig.1) There are other techniques for synthesizing nanoparticles. For example; condensation from a vapor, chemical synthesis and solid-state processes such as mechanical milling. Particles can be coated then with hydrophobic or hydrophilic material, depending on the application.

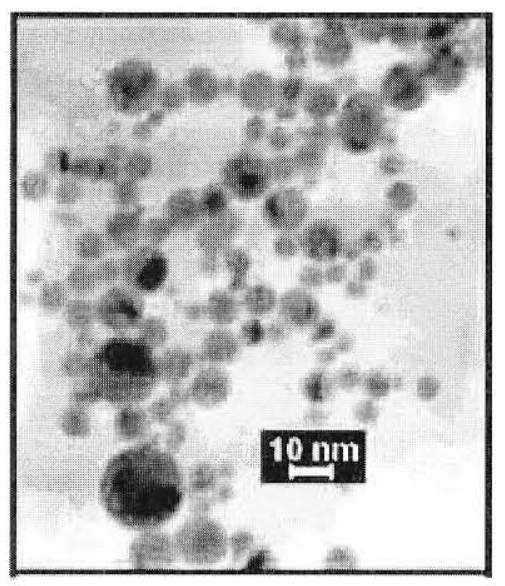

Fig.1 Gold Nanoparticles by laser ablation in aqueous solution of surfactant

The vapor condensation method involves evaporation of a solid metal followed by rapid condensation to form nanosize clusters constituting powder. To obtain metal particles only inert gas is applied, whereas a reactive oxygen atmosphere is applied for metal oxide particles. Another method of the vapor condensation technique is vacuum evaporation on running liquid. This technique utilizes a thin film of viscous material, oil, or a polymer on a rotating plate in a vacuum gauge; the material evaporates or sputters nanoparticles into a vacuum. Another possible method for vapor condensation nanoparticle synthesis is the chemical vapor deposition technique. Both liquid and gas are placed in the reactor and 
the reactor is heated for a specific time depending on the desired shape of the particles. The chemical synthesis technique essentially consists of growing nanoparticles in a liquid medium composed of various reactants. This method is also used to create quantum dots. An example of the solid-state method is milling or grinding to create nanoparticles. The milling time and medium affect the resultant nanoparticles [4].

\section{Nanotubes}

Carbon nanotubes are unique nanostructures with remarkable electronic and mechanical properties. Nanotubes can behave like one-dimensional quantum wires. The structure of a nanotube is a hexagonal network of carbon atoms that has been rolled up to make a seamless cylinder. The cylinder typically has a diameter of approximately one nanometer and length of tens of microns. Each end is "capped" with half of a fullerene molecule. Single-wall nanotubes can be thought of as the fundamental cylindrical structure, and form the building blocks of both multi-wall nanotubes and ordered bundles of single-wall carbon nanotubes called ropes. Many theoretical studies have predicted the properties of single-wall nanotubes. As shown (Fig.2), three types of nanotubes are possible, called "armchair", "zigzag" and "chiral" nanotubes, depending on how the two-dimensional graphene sheet is "rolled up".

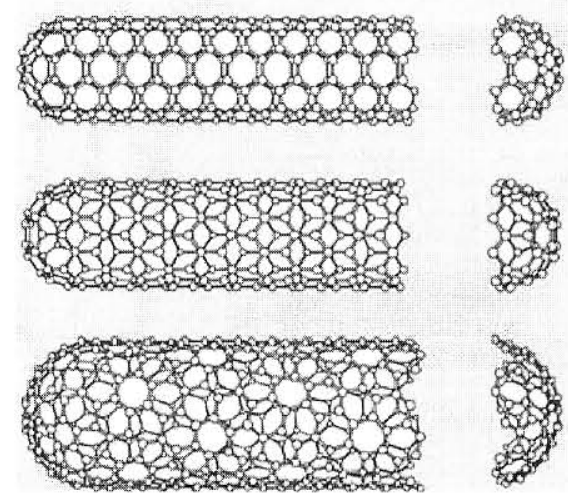

Fig.2 It is shown here a $(5,5)$ armchair nanotube (Top), a $(9,0)$ zigzag nanotube (middle) and a $(10,5)$ chiral nanotube. The diameter of the nanotubes depends on the values of $n$ and $m$. 
The diameter and the chiral angle of the nanotube have been measured with scanning tunneling microscopy (STM) and transmission electron microscopy (TEM). Due to small size of the nanotubes and constant thermal motion of the carbon atoms they have interesting physical properties.

Carbon nanotubes can be prepared by laser vaporization of a carbon target in a tube furnace at $1200{ }^{\circ} \mathrm{C}$. Cobalt and nickel catalyst help the growth of the nanotubes. By use of two laser pulses $50 \mathrm{~ns}$ apart, growth conditions can be maintained over a large volume and for a long time. Flowing argon gas sweeps the nanotubes from the furnace to a watercooled copper collector just outside of the furnace. The result is single wall carbon nanotubes. Recently the carbon-arc method was developed to grow similar arrays of single-wall nanotubes. In this case nanotubes are produced from an ionized carbon plasma, and the heat from the discharge generated by the plasma. At present many international collaborations are working on measuring the properties of single-wall nanotubes. The scanning electron microscopy images of these nanotubes shows ropes of diameters between 10 to $20 \mathrm{~nm}$ and lengths of $100 \mu \mathrm{m}$. These ropes were examined in a TEM, each rope was found to consist of a bundle of single-wall carbon nanotubes aligned along a single direction. The unique electronic properties of nanotubes result from quantum confinement of electrons within the nanotube. Electrons can only propagate along the nanotube axis and their wave vectors point in this direction. The resulting number of one-dimensional conduction and valence bands effectively depends on the standing waves that are set up around the circumference of the nanotube. These simple ideas can be used to calculate the dispersion relations of the one-dimensional bands. 
Calculation of energy dispersion shows that one-third of small nanotubes are metallic and the rest are semiconducting. As the diameter of the nanotube increases there are more wave vectors allowed, thus the band gap decreases. Experiment shows that nanotubes of these different types are stable in pairs. So a metallic carbon nanotube, with semiconducting nanotubes surrounding it can behave like a conducting wire with an insulating shield.

Some of the interesting one-dimensional quantum effects of carbon nanotubes have been observed in Raman spectra, where lattice vibrations are introduced in the material by phonons through the inelastic scattering of light. Dispersion relations have been calculated and shows different vibrational modes coming from carbon atoms; as the nanotube diameter increases more phonon modes appear. Graphite, carbon fibers and carbon nanotubes are expected to be very strong and to resist fracture under extension. Thomas Ebbeson estimated Young's modulus of a carbon nanotube by measuring the vibrations of the free end of a nanotube that was clamped at the other end. Their estimates were consistent with the values of Young's modulus already measured for a graphene sheet. Efforts applying CNTs in electronic devices have been done, for example carbon nanotube field-effect transistors (CNFET) (Fig.3 \&4); CNFET performs with

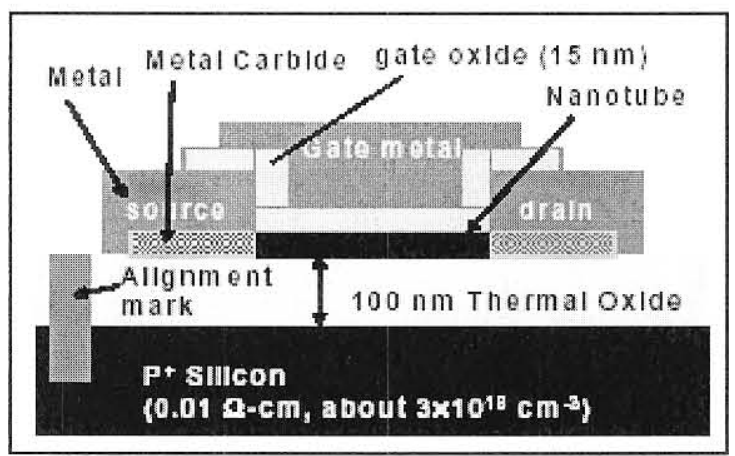

Fig.3 Schematic diagrams of the modeled, single wall carbon field effect transistor[8]

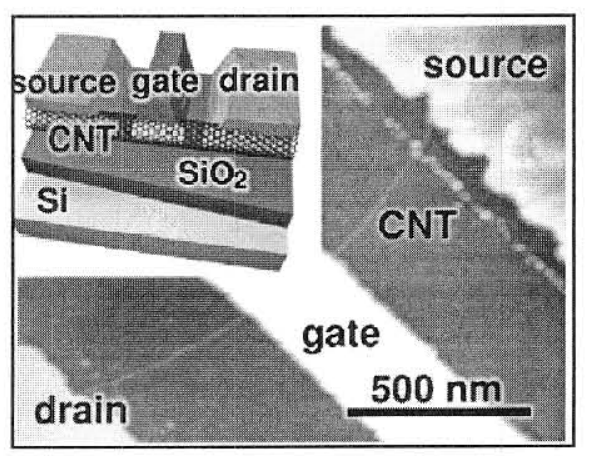

Fig.4 AFM image of CNT in a carbon-nanotube field-effect transistor (CNTFET).

bridging $1 \mu \mathrm{m}$ gape between source and drain [9] 
comparable characteristics of silicon field-effect transistors. CNFETs are Schottky barrier transistors [6]. A composite of SWNT and Polyvinylcarbazole can create a light emitting diode [7]. Research on carbon nanotubes is one of the most popular fields at present and scientists are still characterizing CNTs and trying to use them to make devices. Carbon nanotubes are produced by catalyst driven synthesis by different methods; like arc discharge, Chemical Vapor Deposition (CVD), Pulse Laser Vaporization (PLV) and microwave plasma Chemical Vapor Deposition (PECVD). Catalyst samples prepared in different ways depend on catalyst support, the growth method and on the $\mathrm{pH}$ of the solution. Cobalt and iron were found to be essential in formation of acetylene and other carbon compounds. Iron leads to higher yield and cobalt enhances the quality of CNTs. The resulting CNTs can be SWNTs and MWNTs, grown as bundles or individually [10]. There are reports of carbon nanotubes made from silica, alumina, silicon and metals in a non-crystal structures; they are synthesized by using carbon nanotubes and porous membranes as templates, or by thin-film rolling. These nanotubes, however, are either amorphous or polycrystalline. There are reports about other types of nanotubes. For example single-crystal $\mathrm{GaN}$ nanotubes have been synthesized using hexagonal $\mathrm{ZnO}$ nanowires.

\section{Nanowires (NW)}

Development of nanotechnology and nanoscience has introduced new types of nanostructures called nanowires. NWs appear as semiconductors, carbides, nitrides, oxides, metals and metal oxides. For example GaAs, GaN, GaP, $\mathrm{Si}$ and $\mathrm{Ge}$ nanowires are semiconductor nanosize wire structures, while $\mathrm{GeO}_{2}, \mathrm{SiO}_{2}$ and $\mathrm{Ga}_{2} \mathrm{O}_{3}$ produce oxide NWs and gold, silver and other type of metals make metallic nanowires. Due to their 
small structures they have different properties than material in bulk and have many possible applications. Semiconductor nanowires, like GaAs can be used in FET or metallic NW can be used as tips for scanning tunneling microscopy. Various novel methods have been employed to create NWs.

Scientists have proposed three different approaches for NW growth; Vapor Liquid Solid (VLS), Vapor-Solid (VS), and Solution-Liquid-Solid (SLS) mechanisms. The principle of VLS growth is that the catalyst particle participating in the growth changes to liquid and the vapor phase atoms of the NW-base material saturates the liquid phase (Fig.5). Thus the temperature should not be less than the melting point of the catalyst. Nanowires can grow without a catalyst, for example, in $\mathrm{GeO}_{2}$ nanowire growth. In Vapor-Solid epitaxy the vapor atoms adsorbed to the initial crystalline whisker are only temporarily adsorbed and then diffuse along the lateral surface and reach the tip of the wire to continue the growth. If the vapor atoms don't diffuse and get to the tip they evaporate again.
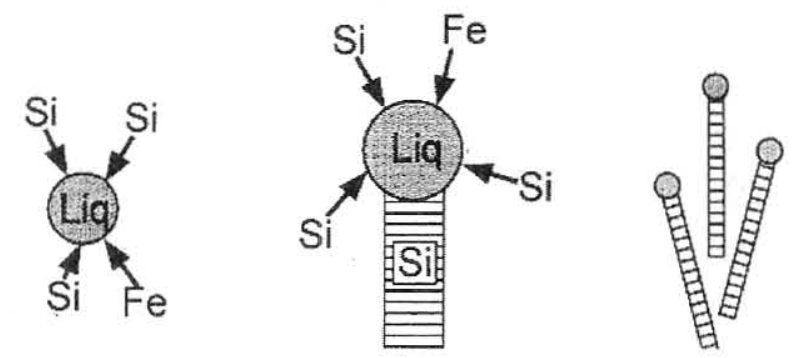

Fig.5 VLS mechanism of $\mathrm{Si}$ Nanowire synthesizes using $\mathrm{Fe}$ as catalyst nanoparticle

There are other ways to synthesize nanowires at lower temperatures ( around $\left.200^{\circ} \mathrm{C}\right)$. An example is the Solution-Liquid-Solid (SLS) method. In this electrochemical approach, a solution containing the nanowire constituent is used with the catalyst on the electrode. The difference between SLS and VLS is, instead of a being in the vapor phase, the atoms 
for nanowires growth contains in the chemical solution. The catalyst remains at the tip of the wire.

Chemical vapor deposition (CVD) applied for thin film growth has been extended to nanowire synthesis. Many types of nanowires have been grown by CVD, for example $\mathrm{Si}$, Ge, GaAs and InP. The growth mechanism can be VLS or VS. In VLS growth the catalyst is attached to the tip of the wire.

Another method for nanowires synthesis is Pulsed Laser Vaporization (PLV). In this technique a hot oven, a pulse laser and a vacuum system are used. This technique is also called Laser Assisted Growth (LAG). The pulsed laser can be either a Nd:Yag, $\mathrm{CO}_{2}$ or an excimer laser to vaporize the target and produce a plume while the growth mechanism can be VLS or VS. The catalyst can be on the target or on the substrate. The laser beam provides two dominant effects in laser ablation; collisional sputtering and thermal sputtering. Collisional sputtering employs the momentum transfer from the incident laser beam to the target material and thermal sputtering is the evaporation of the target by reaching a temperature higher than the melting point of the target.

Vapor Phase Evaporation has been used to synthesize metal and oxide whiskers and then extended to nanowire synthesis. The nanostructures are prepared by physical sublimation of a source material or through reduction of a variety of volatile metal halides. For example $\mathrm{Si}, \mathrm{Ge}, \mathrm{GeO}_{2}, \mathrm{Ga}_{2} \mathrm{O}_{3}$, $\mathrm{GaSe}$ can be synthesized using vapor phase evaporation. The reactor gets heated up and the evaporated or reduced atoms transport in the vapor phase toward the lower temperature gradient and nucleation happen in a random thermal or concentration fluctuation. In Table1 the growth of some nanowires is summarized (there are more possible growth methods for some of these nanowires). 
Electrochemical Deposition or Template Approach is a production method of metallic nanowires synthesis. The synthesis is based on electrochemical deposition on a porous membrane (e.g. polycarbonate membrane). The pores of the membrane get filled with the nanowires so the next step is lift-off and etching of the membrane to release the nanowires [12].

Another remarkable method for nanowires synthesis is Oxide Assisted Growth (OAG). It is a VLS growth using no metal catalyst. Oxygen plays an important role in nucleation and growth of nanowires. The OAG technique allows large quantities of pure nanowires to be produced [13].

\begin{tabular}{|c|c|c|c|c|c|}
\hline \multirow{2}{*}{$\begin{array}{c}\text { Types } \\
\text { of }\end{array}$} & \multicolumn{4}{|c|}{ Growth conditions } & $\begin{array}{l}\text { Average } \\
\text { D of } \\
\text { NWs }\end{array}$ \\
\cline { 2 - 6 } & Source Materials (at\%) & Temp & Atmosphere & Pressure(Torr) & Si \\
\hline & $\mathrm{Si}(95)+\mathrm{Fe}(5)$ & 1200 & $\mathrm{Ar}$ & 100 & $\sim 12$ \\
\hline & $\mathrm{Zn}(50)+\mathrm{Se}(50)$ & 800 & $\mathrm{Ar}+\mathrm{H}_{2}$ & 200 & 60 \\
\hline $\mathrm{SiO}_{2}$ & $\mathrm{Si}(10)+\mathrm{SiO}_{2}(85)+\mathrm{Fe}$ & 1250 & $\mathrm{Ar}$ & 150 & $\sim 15$ \\
\hline & $\mathrm{Ge}+\mathrm{Fe}$ & 900 & $\mathrm{Ar}$ & 150 & $15 \sim 80$ \\
\hline & $\mathrm{Ga}+\mathrm{SiO}$ & 350 & $\mathrm{Ar}+\mathrm{H}_{2}$ & 100 & 60 \\
\hline $\mathrm{ZnO}$ & $\mathrm{Zn}+\mathrm{Se}$ & 200 & $\mathrm{Ar}+\mathrm{H}_{2}$ & 200 & 40 \\
\hline
\end{tabular}

Table1

The nanowire diameter ranged from $5 \mathrm{~nm}$ to $500 \mathrm{~nm}$ depending on the material and the length varies between $1 \mu \mathrm{m}$ to $100 \mu \mathrm{m}$. The morphology of NWs can be crystalline, poly 
crystalline and amorphous. Semiconductor nanowires can be made from one element, for example from group IV (Si, Ge) or it can be a multi compound, for example binary group III-V (GaAs, GaP, InAs and InP), binary group $\Pi$ - VI (ZnS, ZnSe, CdS and $\mathrm{CdSe})$ on ternary III-V $(\mathrm{GaAs} / \mathrm{P}, \mathrm{InAs} / \mathrm{P})$ [14]. Superlattice nanostructures in the form of heterostructures have been synthesized from group III-V and group VI in a uniform, crystalline structure.

The results of the VLS growth process for synthesizing varieties of semiconductor nanowires depend on the Pseudobinary phase diagram of the reactant chemicals (Fig.6). The percentage of the catalyst to the material is thereby defined. For example for GaAs laser assisted growth using $\mathrm{Au}$ as the catalyst, it is revealed that the atomic percentage of GaAs to $\mathrm{Au}$ should be $(\mathrm{GaAs})_{0.95} \mathrm{Au}_{0.05}$. Also, phase diagram helps to approximately determine the synthesis temperature range.

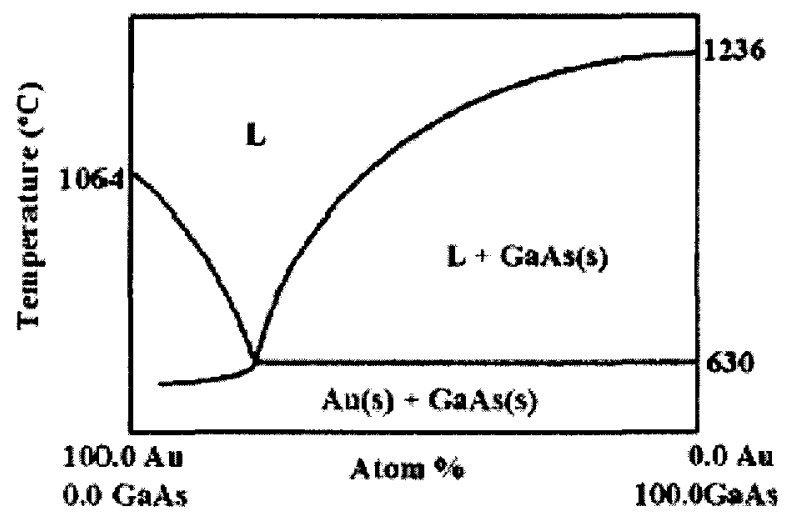

Fig. 6 Pseudobinary phase diagram for $\mathrm{Au}$ and $\mathrm{Ga} \mathrm{As}$

The resulting NWs synthesized by the LAG method can be as small as $3 \mathrm{~nm}$ in diameter and about $10 \mu \mathrm{m}$ long. High resolution microscopic images from a SEM or TEM reveal that the catalyst particle is attached to the tip of the NW and Scanning Tunneling 
Microscopy (STM) or Field Effect TEM (FETEM) show the crystalline structure of the nanowires and the uniformity of the atoms in the structure. (Fig.7)

Super-lattice nanowires are essentially heterostructures of two types of semiconducting crystalline structures. Composition of modulated superlattices of $\mathrm{GaAs} / \mathrm{GaP}$ nanowires
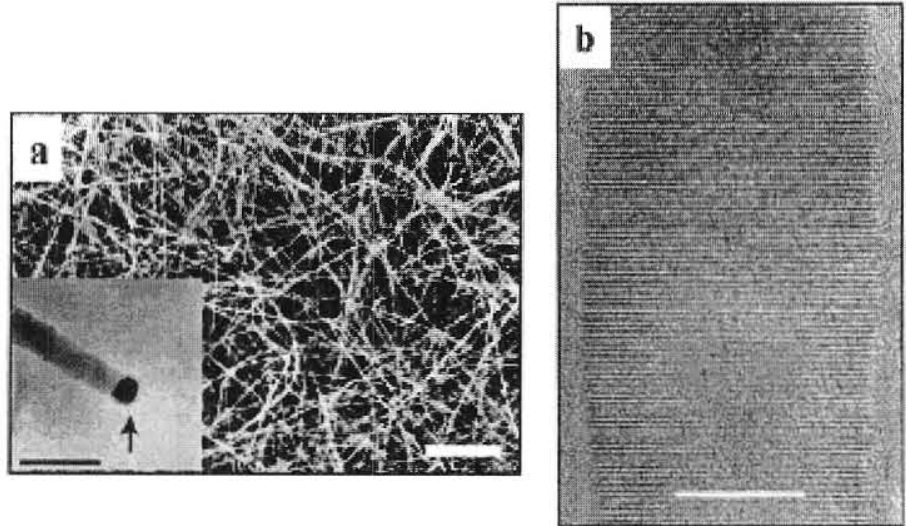

Fig.7 (a) Low resolution TEM image of GaAs Nanowires, the catalyst particle is attached to the tip of the NW, (b) FETEM image of the crystalline structure of GaAs Nanowire growth by VLS method

have been synthesized (Fig.8). Furthermore, In As/InP, n-Si/p-Si nanowires and n-InP/pInP have been synthesized. The growth happens by alternating the vaporization between two different materials and changing the vapor-phase reactant material in a specific period of time. These novel structures composed of nanowires can have unique photonic and electronic properties and the potential applications includes nanosize LEDs and nano-barcode.
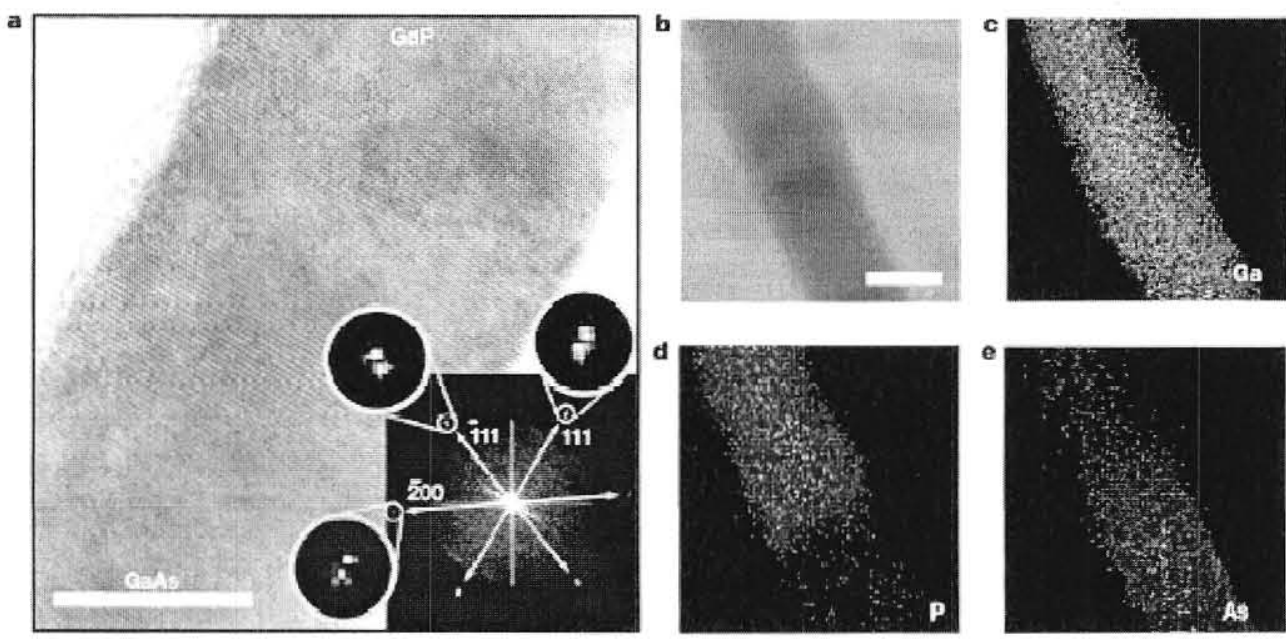

Fig.8 (a)GaAs/GaP nanowire TEM image and the diffraction pattern (b),(c),(d) and ( e)
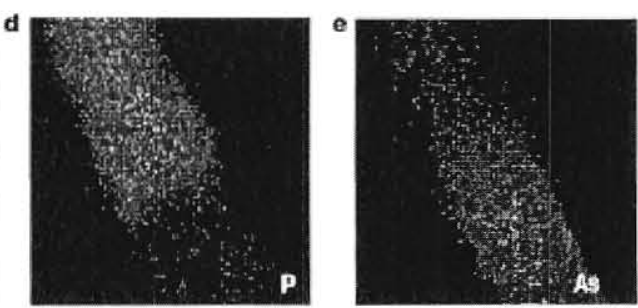
EDS result for NW, the border of the chemicals is very clear and sharp 
There are other types of nanostructures such as nanoribbons, nanorods and nanobelts, which are subgroups of nanowires and we will be covering later in chapter III.

\section{Nucleation}

Nucleation is an important concept in the growth process to produce new particles from solution. There are three kinds of nucleation; homogenous nucleation, heterogeneous nucleation, and secondary nucleation.

Homogenous nucleation happens in the absence of a solid-phase. The "Lamer's Model" describes the nucleation process of sulfur sols and can be applied to other chemicals as well. In this model, when a solution gets supersaturated a new phase nucleates out of it. The particles grow by molecular addition and decrease the solution concentration, when the concentration drops below a critical level nucleation stops and particles continue to grow by molecular addition until reaching the equilibrium concentration of the participant species. In a short period of nucleation all particles reorganize through a selfsharpening process in which the bigger particles do not grow and the smaller ones grow faster. The particles aggregate with other particles and produce bigger particles when the solute is deposited on the solid surface.

Fig.9 Schematic of the formation of monodepressive spherical particles by nucleation and aggregation
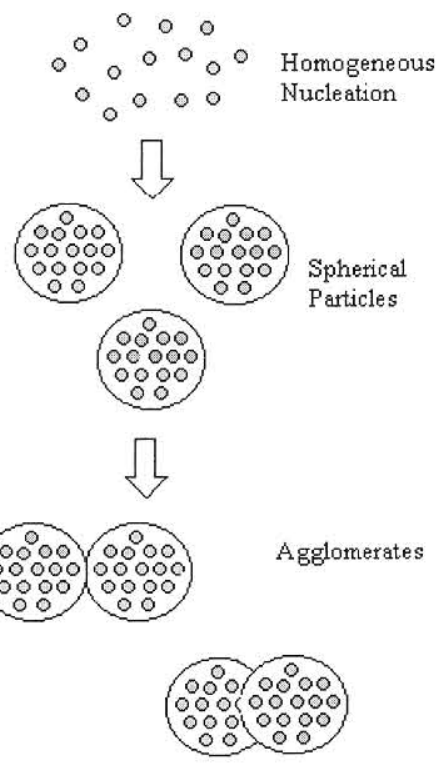
In the aggregation model the primary particles grown are unaware of the presence of other particles then the particles aggregate and produce bigger particles and decrease the suspended particles in the solution. The aggregated particle grows bigger by combining with the unstable smaller particles. (Fig.9) Nucleation on the solid is heterogeneous. In the nucleation process a critical cluster is formed for a stable crystal growth.

\section{Chemical Vapor Deposition}

In chemical vapor deposition (CVD) the precursor is introduced to the reactor in the vapor phase and the molecules adsorb onto a substrate held at higher temperature. These adsorbed molecules thermally decompose or react with other gases (vapors). The grown materials can be metals, semiconductors, or ceramics and can be in amorphous, polycrystalline or crystalline form.

Thermodynamically, the CVD process requires high temperatures and low pressure. In principle, CVD consist of three steps, (1) diffusion of reactant to the growth surface through a boundary layer (2) chemical reaction on the growth surface and (3) removal of the gases produced by the reaction at the growth surface. The concept of boundary layer and diffusion resistance depends on time and distance and is defined by the mass-transfer coefficient. If the mass- flow coefficient of the reactants is less than the product, it is a kinetically controlled system, otherwise it is diffusion controlled. Diffusion mostly happens at high temperature when a homogenous nucleation occurs and kinetic effects occur at low temperature and high flow rate. Homogenous nucleation happens when the 
reactor is highly heated and the reactant material is in the gas-phase and it diffuses onto the surface as small solid particles and growth occurs. The growth rate depends on the diffusion coefficient of the solid particles. Pressure is another parameter of growth rate; it does not affect the mass transfer rate but higher pressure increase reaction rate.

Chemical vapor deposition can be carried out at atmospheric or low pressures. Low pressure CVD is used to reduce the gas-phase nucleation and leads to a thin film of the desired material. Nucleation in the gas phase can lead to unwanted particles in the synthesis. However, particle formation in the gas phase can be used to synthesize nanoparticles under some special conditions. So controlling particle formation in the gas phase and controlling the pressure according to the particles' size are key steps for the growth of nanostructures.

Three different growth modes result from nucleation on a substrate. If the substrate and deposited film have a little mismatch (less than $0.2 \%$ ) a layer-by-layer growth results because of the higher surface energy of the film than the substrate. But at lower film surface energy, the result is partly layers of crystalline planes and partly clusters- looking islands. At lower encrgy the growth looks like the clusters of material.

There are different types of CVD, for example Plasma enhanced CVD (PECVD) or Low Pressure CVD (LPCVD) or Filament CVD. A CVD apparatus consist of three parts. The precursor sources can be solid or liquid with a bubbler and a carrier gas or it can be a gas source. The gas flow is controlled with a gas flow controller. Next is a heat source like an internal resistance heater or external heater.

\section{Physical Vapor Deposition}


Deposition of films by condensation of the vapor phase is commonly called physical vapor deposition (PVD). Some examples of PVD are sputtering, electron-beam evaporation, thermal evaporation, arc-plasma deposition, pulse laser deposition and more. These techniques have been using for deposition of different layers for electronic purposes. Recently they have been used for nanostructure synthesis, especially for thin films.

Sputtering is a method used to get multilayer structures of different types of materials. The sputtering system consists of a vacuum chamber (between $1-100$ mtorr), an inert gas supplied to the chamber and a RF or DC voltage applied between cathode and anode. The gas gets ionized, producing plasma that bombards the target with the sputtered particles. Some types of sputtering systems have a magnetron to direct the plasma to the substrate. Pulsed laser deposition is another known method for PVD which is used for thin film deposition, nanowires synthesis and more. Various materials can be deposited as thin films such as metals, semiconductors, nitrides, oxides and organic/polymer compounds. Pulsed laser deposition is a process which directs a high-power laser onto a solid target and directs the vaporized material toward the substrate in a vacuum chamber.

\section{Laser Ablation (Pulse laser Vaporization)}

The laser ablation method is a promising technique used to synthesize nanostructures mostly in the crystalline phase. Laser ablation has many advantages compared to other methods: the high energy laser beam can be operated in pulsed, continuous mode and different wavelengths of laser (depending on the laser type) may be obtained by using some simple optics. Ablation creates a large plume of evaporated material that can be a 
mix of different chemicals from a multi-component target. Ablation can be controlled by the spot size, laser energy and wavelength. In Fig.10 the schematic of a laser ablation system is depicted.

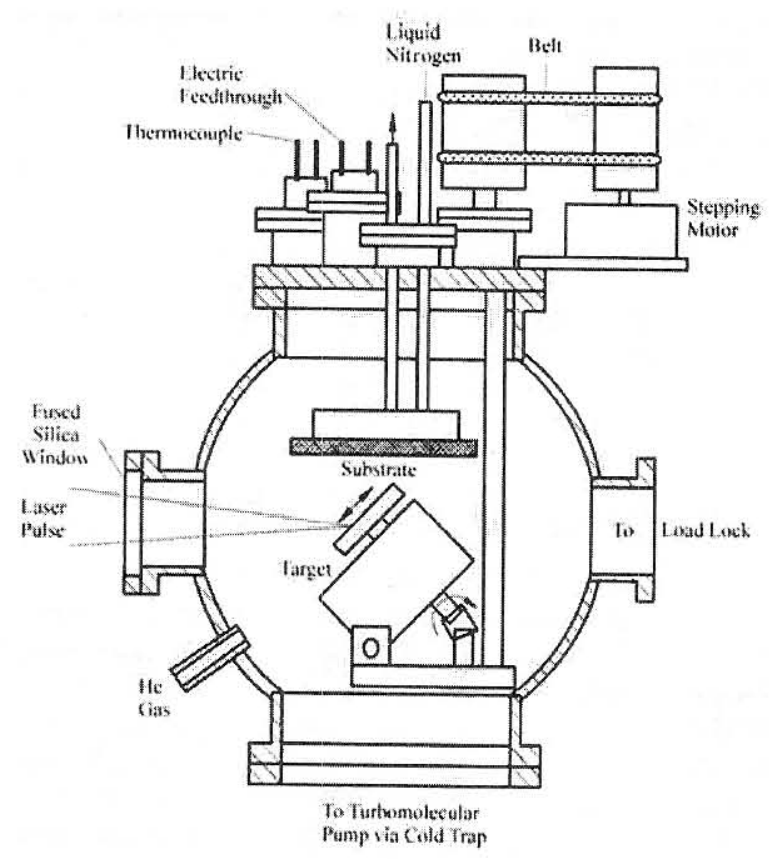

Fig.10 Laser ablation system

The laser beam affects the target in three possible ways, photo thermal ablation, plasmaassisted ablation and photochemical ablation, depending on wavelength, energy and pulse duration of the laser. In the thermal process the photons' energy is absorbed by electrons in the target and converts it to an oscillating energy and increase in temperature. The target is cooled by evaporation. In the second path, plasma-assisted ablation, high peak power in the laser pulse produces plasma next to target. High-energy ionized gas bombards the target, then target atoms are heated and ejected from the target. Photochemical ablation is a quantum phenomenon. If the laser energy is of the same 
order as the bonding energy of the molecules it is absorbed and stored by molecule. This leads to breaking the bonding and ejecting the material.

The physical model of laser ablation consists of a high-energy laser beam impacting the target converted to heat, and vaporizing the target. The vapor is partially ionized. This plasma condenses and produces clusters that migrate to the cool part of the reactor and are collected there. Synthesis results can be thin films, particles or wire-like structures in crystalline or amorphous structural form. [15]

Pulsed laser vaporization (PLV) is a combination of the laser ablation method and vaporliquid-solid (VLS) growth mechanism ( also called Laser Catalyst Growth, LCG) . A PLV system consists of a laser, tube furnace, vacuum pumps and gas introduction system. Fig.11 depicts a schematic of a PLV system.

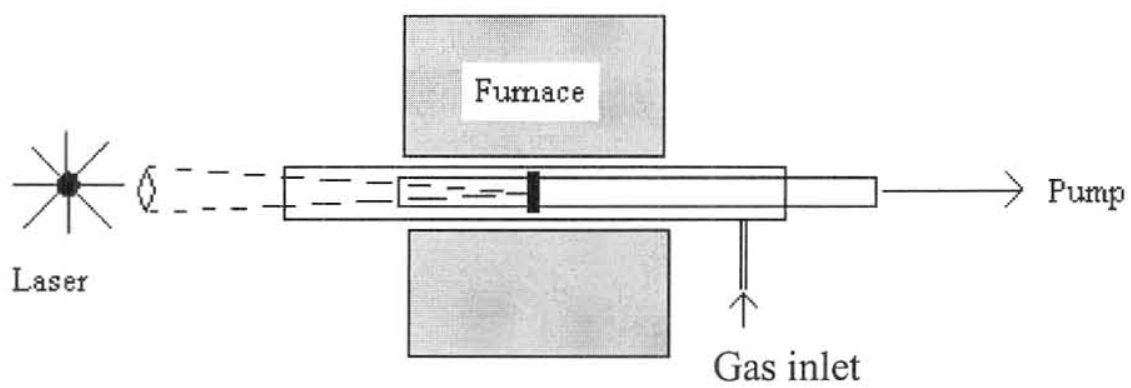

Fig.11 PLV system

In this method catalytic particles play an important role in the growth mechanism. In VLS growth a liquid metal cluster or catalyst acts as the energetically favored site for absorption of gas-phase reactants. The cluster supersaturates and grows one-dimensional structure of the material. The approach results in single crystalline wirelike structures with diameters ranging $5 \mathrm{~nm}-100 \mathrm{~nm}$ and of tens of micrometers length. [16] A key feature 
in VLS growth from LCG procedure is the equilibrium diagram (or Pseudobinary phase diagram), which predicts catalysts and growth conditions. Single and multi-component semiconductors, oxides and carbon nanotubes have been synthesize using the PLV method. Nanowires of the binary group III-V materials (GaAs, GaP, InAs) and binary II VI compounds ( $\mathrm{ZnS}, \mathrm{ZnSe}, \mathrm{CdS})$ have been produced [17]. Fig.12.a shows silicon nanowires grown by the LAG method, using a $\mathrm{KrF}$ excimer laser and target of $\mathrm{SiC} / \mathrm{SiO}_{2}$ [18].

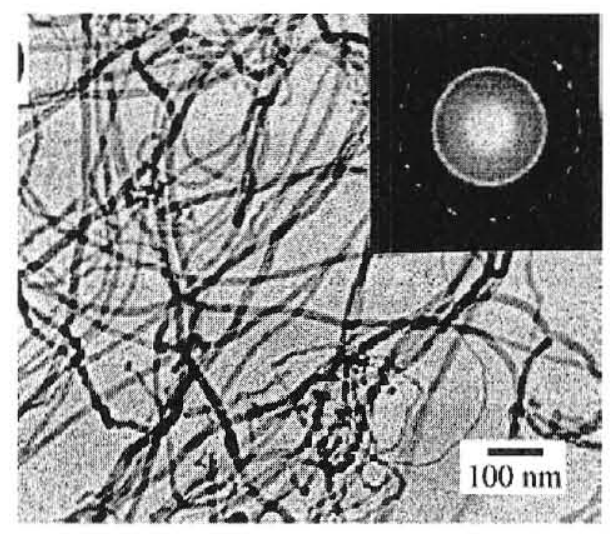

Fig.12 Laser assisted growth result of $\mathrm{Si}$ nanowires

\section{Transmission Electron Microscopy (TEM)}

TEM is a powerful technique for structure characterization with real space imaging and resolution of $1 \mathrm{~A}^{\circ}$ and can provide quantitative information as well as chemical analysis. . The transmission electron microscope (TEM) operates on the same basic principles as the light microscope but uses electrons instead of light. What you can see with a light microscope is limited by the wavelength of light. TEM uses electrons as the light source and their much shorter wavelength provides resolution of thousand times better than that with a light microscope. The electron transmits through the sample and reveals the finest structures. Electrons have advantages as do X-ray, because they can be focused easily and are more flexible for imaging. In high-resolution transmission electron microscopy 
(HRTEM) the phase of the diffracted electron wave is preserved and interferes with the phase of the transmitted wave. An electron gun at the top of the microscope emits electrons that travel through vacuum in the column of the microscope. Instead of glass lenses focusing the light in a microscope, the TEM uses electromagnetic lenses to focus the electrons into a very thin beam. The electron beam then travels through the specimen. Depending on the density of the material present, some of the electrons are scattered and disappear. At the bottom of the microscope the unscattered electrons hit a fluorescent screen, which gives rise to a "shadow image" of the specimen with its different parts displayed in varied darkness according to their flux density [19]. A schematic of a TEM is shown in Fig.13.
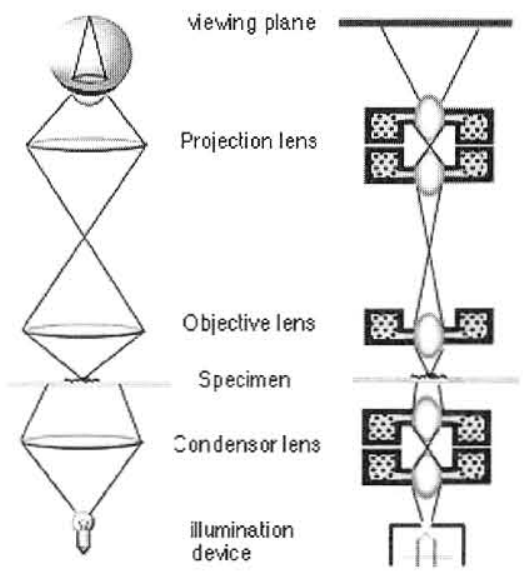

Fig.13 Schematic of optical and Imaging of the TEM system

TEM operate based on electron scattering. The electron beam illuminates a thin specimen and according to the atom-electron interaction, different features happen which can be measured. High-energy electrons $(\sim 20 \mathrm{KeV})$ impact single atoms elastically or inelastically. Elastic scattering conveys the image and inelastic interaction results in Xray or Auger electron emission which can give additional information. The electron beam is converged and controlled by electromagnetic lenses. The image can be recorded on 
film or CCD detector. Fig.14.b shows some images taken by TEM [20]. High resolution TEM images are good for studying crystalline features in a material, determining the defects in a crystal, imaging the atomic arrangement at interfaces and investigating the crystalline arrangement of an unknown material.
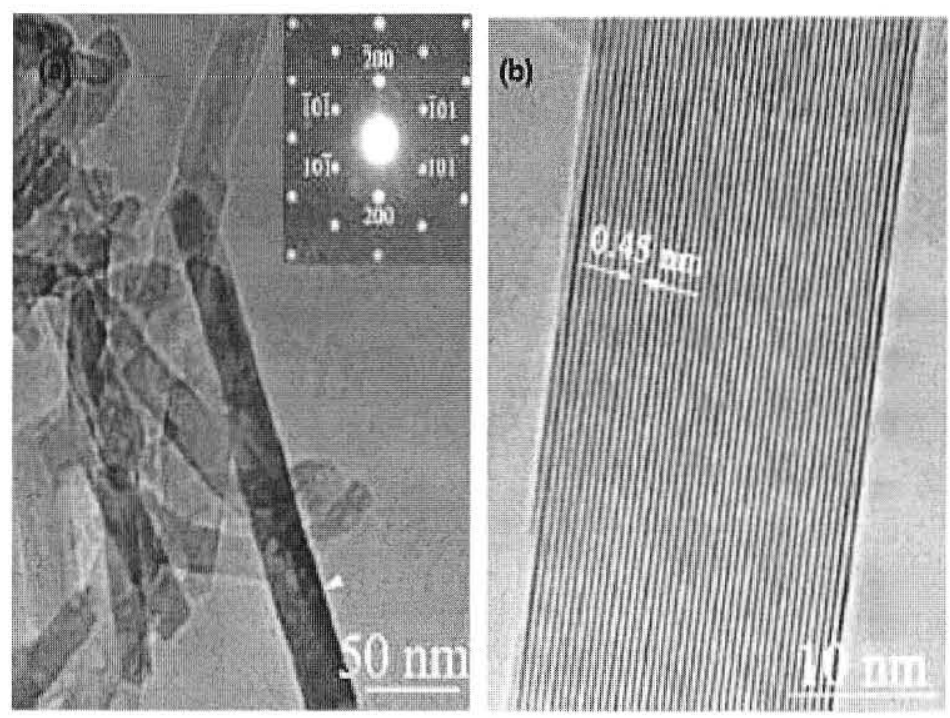

Fig.14 TEM image of some dispersive Boron NWs. (a) the corresponding SAED pattern of the arrowed boron nanowire.

(b) HRTEM image of the arrowed boron nanowires

Selective area diffraction (SAD) is another feature used in TEM. The region of interest is determined by imaging the specimen and a $\mathrm{SAD}$ aperture is used to image only that region. A diffraction pattern of elastically scattered electrons appears on the viewing screen. Convergent beam electron diffraction is an enhanced mode of SAD, which confines the electrons zoned area to approximately two nanometers for nano-diffraction. The result appears as bright spots for crystals and eccentric bright rings for poly-crystals. The separation of diffraction spots on the viewing screen determines the interplanar spacing of the crystal. Fig.14.a shows a SAD pattern for boron nanowires.

\section{Scanning Electron Microscopy}


Scanning electron microscopy is another technique used for imaging small structures. In the SEM, the image is formed by a very converged and high-energy electron beam, which is focused on the surface of the specimen. The beam is scanned over the specimen in a series of lines and frames called a raster. The raster movement is accomplished by means of small coils of wire carrying the controlling current, causing electromagnetic deflection. A schematic drawing of an electron microscope is shown in Fig.15. The specimen is bombarded with electrons over a very small area. The electrons may be elastically reflected from the specimen, with no loss of energy. They may be absorbed by the specimen producing secondary electrons of very low energy, together with X- rays. They may be absorbed and give rise to the emission of visible light (an effect known as cathodoluminescence). And they may give rise to electric currents within the specimen. All these effects can be used to produce an image. By far the most common, however, is image formation by means of the low-energy secondary electrons [21].

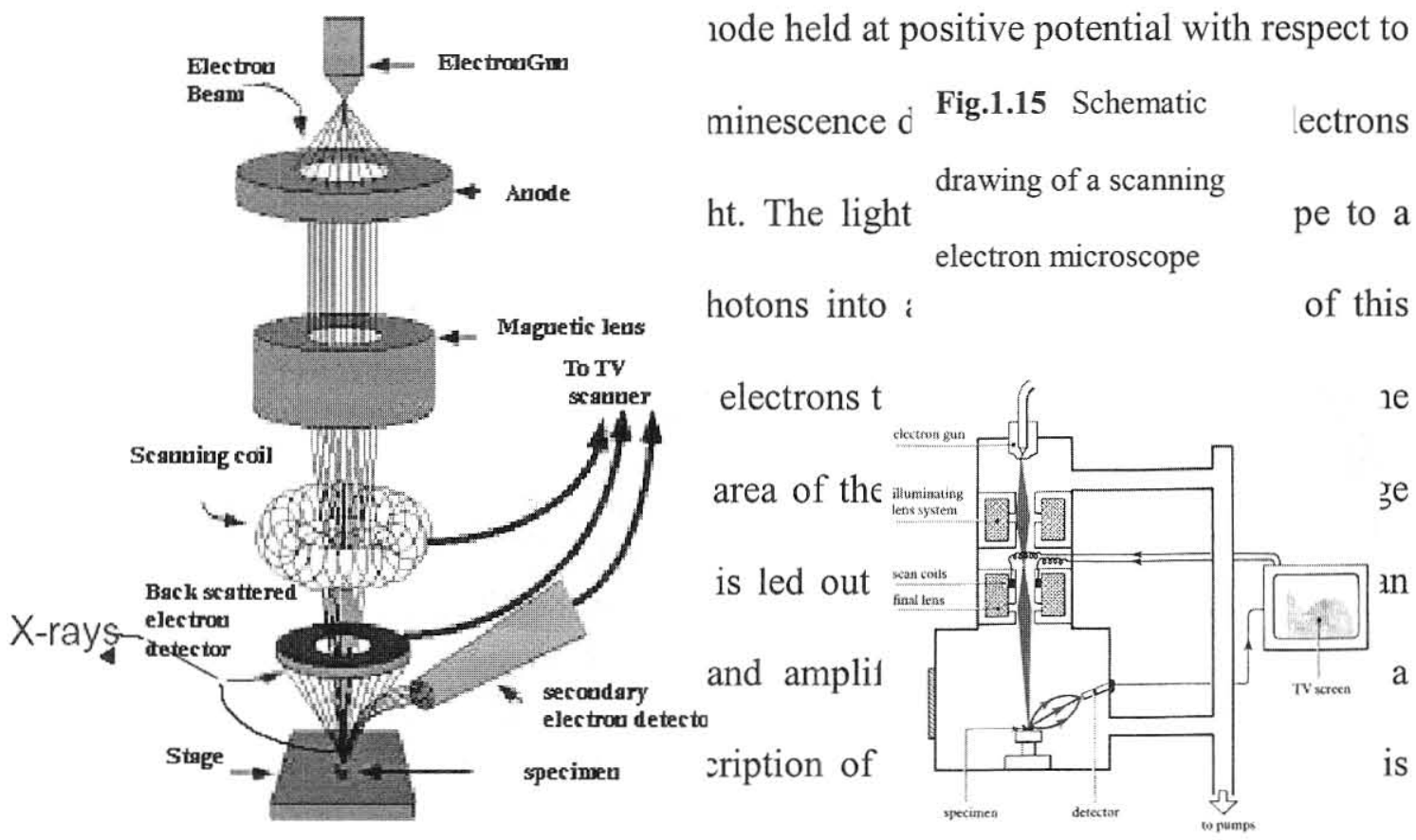

equany appıcabıe to elasıcany scatrerea eıectrons, X-rays, or photons of visible light - 
except that the detection systems are different in each case. Secondary electron imaging is the most common because it can be used with almost any conducting specimen. X-ray analysis equipment as a bolt-on accessory is possible as well. A Secondary electron is an electron that is weakly bound to the sample and is ejected with at most tens of electron volts and can travel a short distance through the material (less than $100 \AA$ ), Therefore

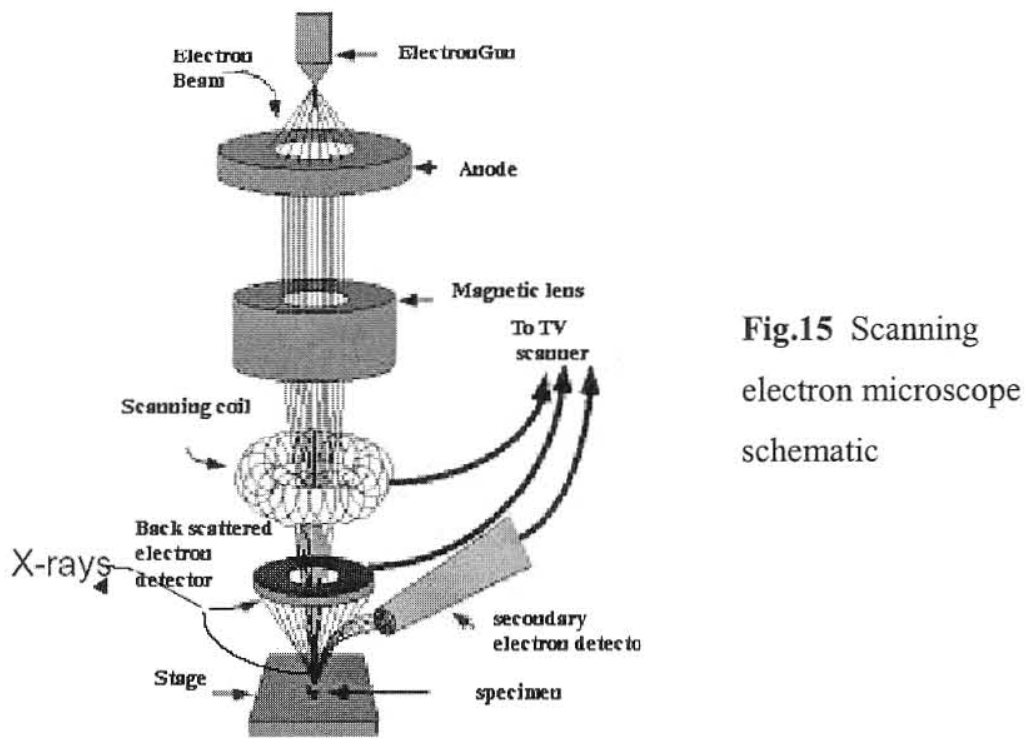

secondary electrons come from near the surface and they are highly interesting for surface topography, being more likely to emerge from the peaks rather than valleys.

\section{X-Ray Powder Diffraction}

A conventional $\mathrm{x}$-ray tube consists of a vacuum tube diode $(\mathrm{Mo}, \mathrm{Co}, \mathrm{Cu}, \mathrm{Fe} . .$.$) and a$ high voltage filament for energetic electron emission used to excite the material for powerful $\mathrm{x}$-ray production [22]. The emitted $\mathrm{x}$-ray is characterized as a $\mathrm{K} \alpha \mathrm{x}$-ray for transition of electrons from shell $\mathrm{L} \rightarrow \mathrm{K}$ and $\mathrm{K} \beta$ for transition from $\mathrm{M} \rightarrow \mathrm{K}$ shell, (x-ray 
emission happens when an energetic electron impacts the material and excites inner shell electrons, then outer shell relax and fill the vacancy, releasing an x-ray), (Fig.16).

In a diffraction experiment, the incident wavelength must be comparable to the spacing between the atoms. Bragg's law (Eq.1) explains the diffraction pattern and predicts the lattice spacing of incoming waves to two crystalline planes in sequence, with lattice

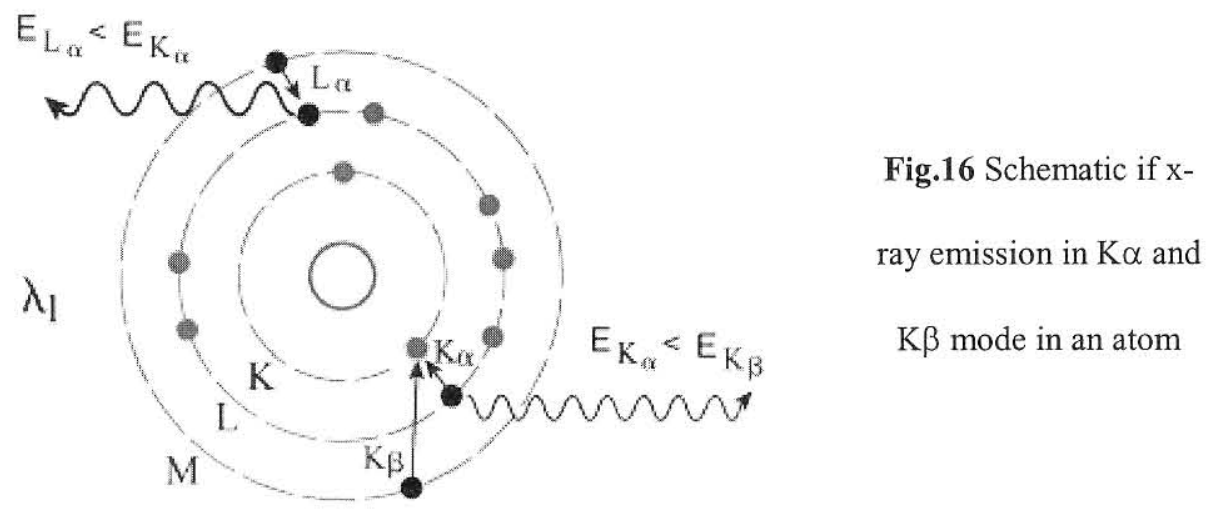

spacing $d$, incident angle $\theta$ and $\mathrm{x}$-ray wavelength $\lambda$.

$$
2 d \sin \theta=n \lambda
$$

The interference pattern of the scattered beams is detected. The diffraction pattern from a material contains many distinct peaks, each corresponding to a different interplanar spacing $d$, which varies according to the particular crystalline structure. In XRD data a constructive interference pattern forms a corresponding peak (Fig.17). The intensity of the peak varies for different interplanar spacing and each diffraction angle has corresponding Miller indices (hkl). 
There are many individual crystals of random orientation in a XRD sample, so all possible Bragg's diffractions can be observed in the powder pattern. For simple crystals, it is usually possible to guess the structure from XRD books; more complicated XRD systems require use of computer to investigate the structures. If the material has a composition of more than one crystalline phase, it can be indexed using phase diagram handbooks. Strain effects in the crystals can shift the x-ray diffraction peaks, and these shifts can be calculated. A distribution of strain and inhomogeneous chemical composition causes broadening of the peaks [19].

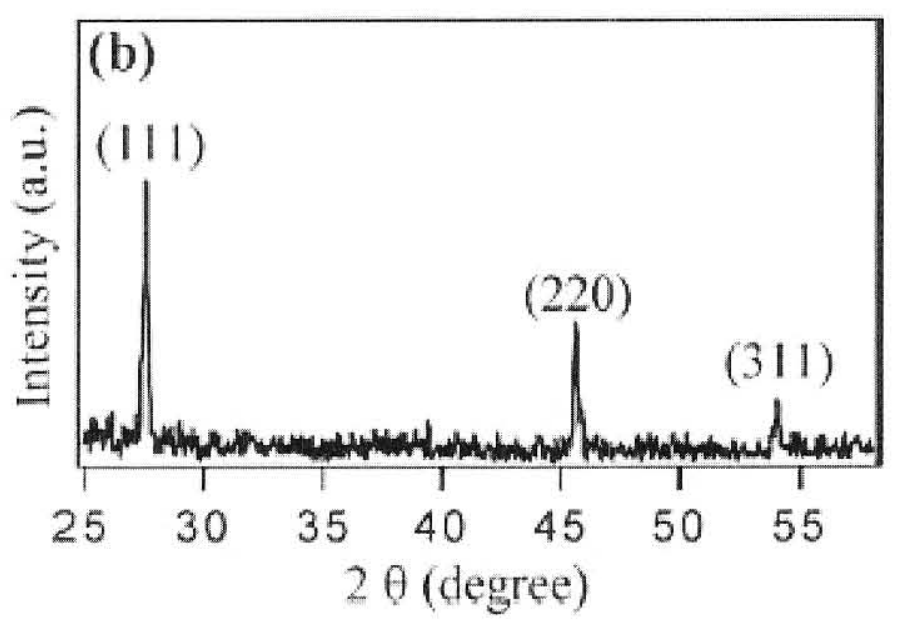

Fig.17 XRD spectrum for GaAs nanowires[23].

\section{Energy Dispersive X-ray spectroscopy}

Inelastic high-energy electron scattering can cause the emission of $\mathrm{x}$-rays from the specimen, which is called energy dispersive $\mathrm{x}$-ray spectroscopy (EDS). EDS is a chemical microanalysis technique performed in conjunction with SEM or TEM, with microscopes fitted with an x-ray detector and photo-multiplier. When the sample is bombarded with electron beam, electrons are ejected from the core level of the atom and leave a vacancy. Electrons from higher shells fill the electron vacancy and release $\mathrm{x}$-rays. 
The EDS $\mathrm{x}$-ray detector measures the number of emitted $\mathrm{x}$-ray versus their energy. The $\mathrm{x}$ ray energy characterizes the emitting element, (Fig.18).

X-ray detection of elements from beryllium to uranium is possible, with an accuracy of 0.1 weight percent. The electron beam scans the selected area and $\mathrm{x}$-rays are detected for discrete positions along the line, which is called a line profile analysis. It is possible to map the elemental distribution at a specific area; the map is recorded using image density.
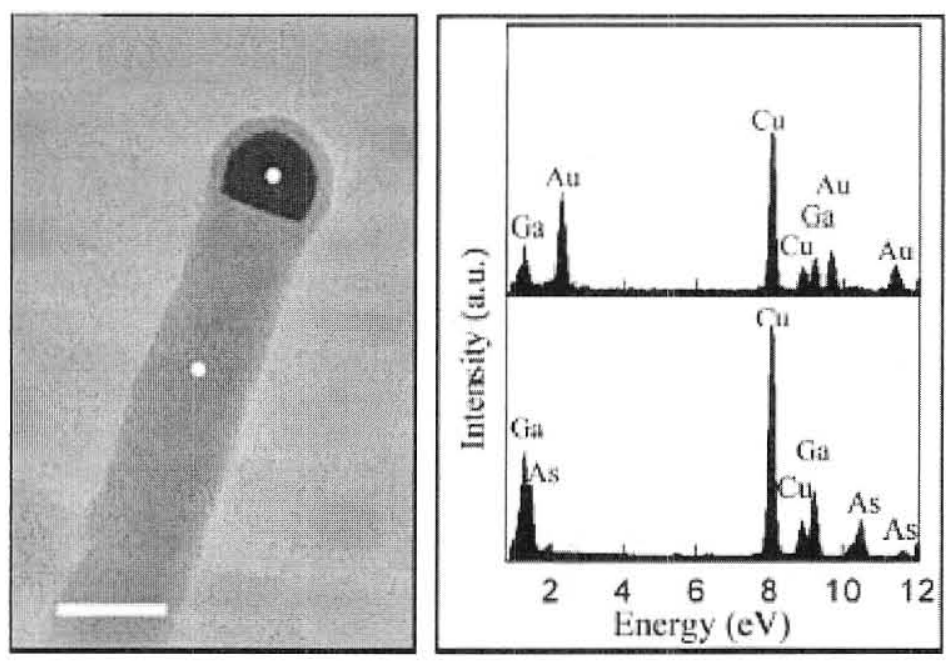

Fig.18 TEM image of the end of GaAs nanowires (the dark part at tip of wire is gold as catalyst) and EDS spectrum for two white spots on the image [25]

\section{Raman Spectroscopy}

Raman spectroscopy is used to study vibrational, rotational, electronic, and other frequency phonon modes in a material. It relies on inelastic scattering of an optical phonon from the lattice structure of the material and creates or annihilates optical photons. Usually a monochromatic laser in the visible, UV or IR range illuminates the sample, resulting in emission or absorption of a phonon shifting the frequency of the 
outgoing laser beam. The shift in energy gives information about the phonon modes in the system; a phonon is the quantized vibration in a crystal structure. The Raman scattering is weak and it is important to separate it from the laser scattered light. The spectrometer of a Raman system consists of a holographic diffraction grating and multiple dispersion stage to obtain a high rejection of the laser.

A photo-multiplier (PMT) or a CCD detector detects the scattered photons. In (Fig.19) the principle of Raman spectroscopy is shown.

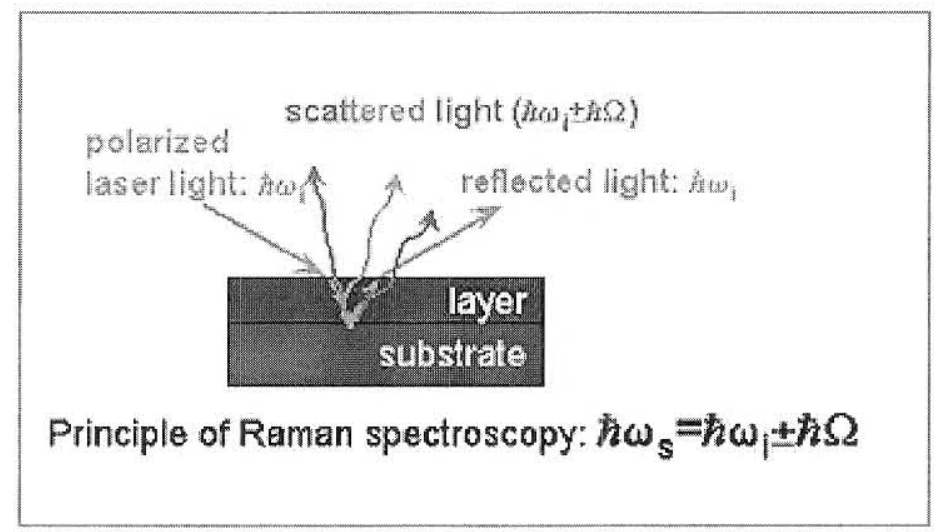

Fig.19 Raman scattering

The photons are scattered by the emission or absorption of phonons, shifting photon energy which is measured by the interferometric technique. One phonon contributes to the scattering of a photon and changes the photon frequency to $\omega_{\mathrm{s}}(\mathrm{k})$. The process is called "Brillouin scattering" when the photon emitted or absorbed is acoustic, and Raman scattering when it is optical. The Raman signal gives information on the population of a given phonon mode in the ratio between the Stokes (down-shifted) intensity and antiStokes (up-shifted) intensity. When a photon is scattered elastically from a molecular 
system and the emitted photon has the same wavelength as the absorbing photon it is called "Rayleigh" scattering. In Raman scattering the energy of the incident and scattered photons are different. The energy of the scattered radiation is less than the incident radiation for the "Stokes" line and the energy of the scattered radiation is more than the incident radiation for the "anti-Stokes" line (Fig.20) The intensity of anti-stokes scattering is much less than stokes.

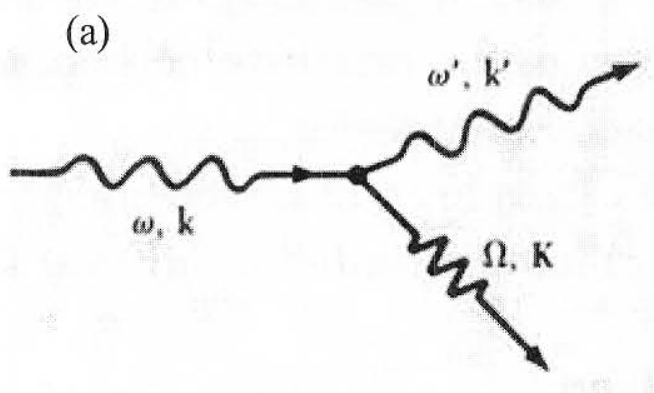

Stokes

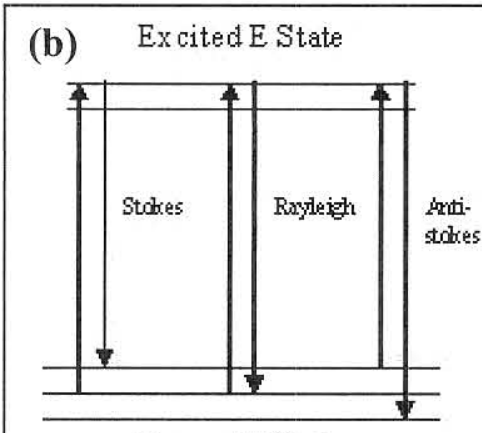

Ground E State

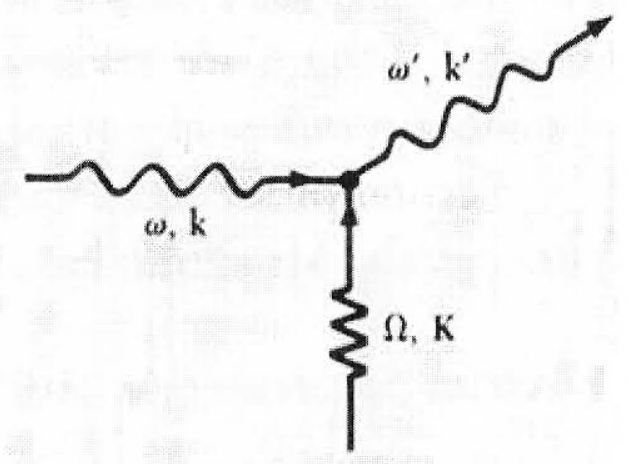

Anti-Stokes
Fig.20 (a) Raman excitation (b) Stokes and anti-stokes and Rayleigh Radiation 


\section{CHAPTER II}

\section{EXPERIMENT}

\section{Synthesis}

In this chapter two different synthesis methods and the growth result will be discussed. CVD and PLV are the approaches that have been tried for growth of various structures of different materials in the lab. The growth process is very sensitive so small changes of any parameter can produce a new type of nanostructure. Following are details of the experimental methods and results:

\section{Design and set-up of the chemical vapor deposition system}

As mentioned in "chapter I", a chemical vapor deposition is a versatile way of producing nanostructures. The body of the CVD consists of four parts; the reactant chemical inlet, reactor, furnace and exhaust. The chemical was introduced in gas phase through a valve to the five-channel mass flow controller (EDWARDS, Model 825).

Mass flow controller is a combination of flow transducer and flow controller, which measures the mass flow rate of the gas flowing through the hose and controls the gas flow with an internal servo amplifier and proportional control valve. Depending on the reacting materials, up to five gases can be mixed and introduced to the reactor simultaneously. The reactor is a quartz tube, with $3.80 \mathrm{~cm}$ outer diameter and $61 \mathrm{~cm}$ length, and is attached at both sides with QF quick connectors, which is connected to QF 
flange with five swagelok gas inlet form one side and one swagelok for outlet on the other side for the exhausts. One zone tube furnace (Barnstead International, model F79300) used for heating the reactor The furnace power and the set point is controllable and provides enough flexibility of temperature ramping for any set point up to $1200{ }^{\circ} \mathrm{C}$. The last part is the exhaust, which starts from the outlet swagelok to a tygon tube with its end immersed in a beaker of water. This provides backpressure, which helps to control the reaction pressure easier.

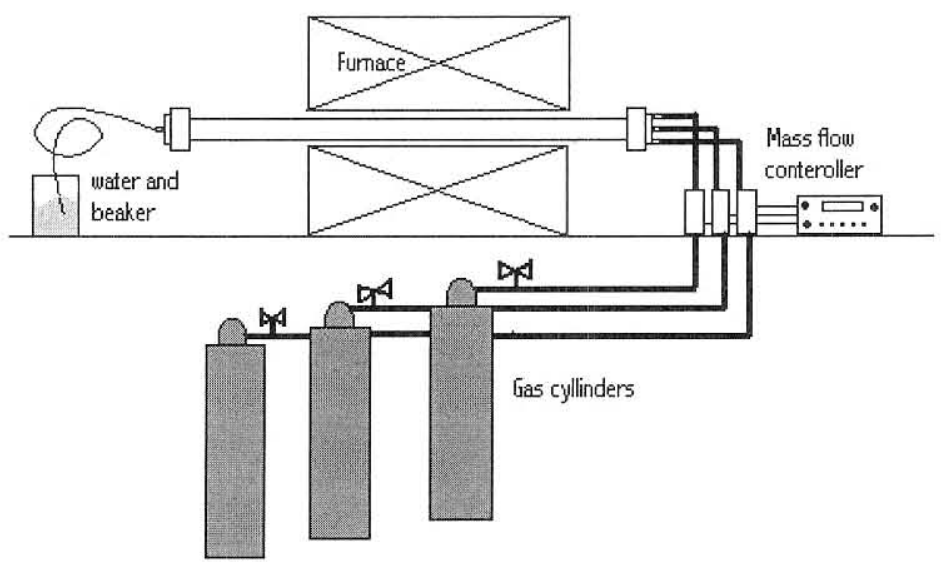

Fig. 21 CVD schematic

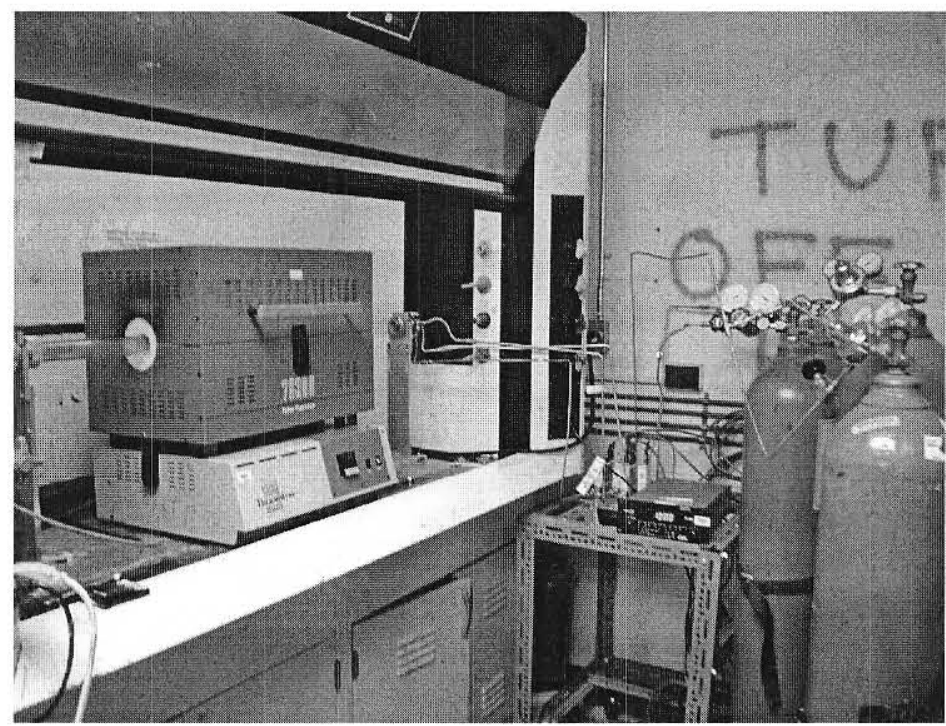

Fig. 22 CVD set up in the laboratory 
The bubbles of the gas coming out of the tube into the water helps to make sure the gas is flowing with a glance. In Fig. 21 the schematic of the CVD is shown and in Fig.22 the actual set up is depicted.

\section{CVD growth of Gallium oxide}

In this section the synthesis process of gallium oxide $\left(\mathrm{Ga}_{2} \mathrm{O}_{3}\right)$ using CVD system is presented. An unsuccessful attempt to grow gallium arsenide nanowires produced unique $\mathrm{Ga}_{2} \mathrm{O}_{3}$ nanostructures. This reaction was done in CVD system but by physical vapor decomposition (PVD) method.

The raw material was a GaAs n-type wafer, doped with Tellurium, (University wafer Inc.), which was ground carefully and mixed with gold (atomic percentage GaAs: Au was 0.95: 0.05). The mixture was placed in a quartz boat in the middle of the tube furnace inside the quartz reactor; argon gas was passed through the reactor during the process and the flow rate was kept at $100 \mathrm{sccm}$ (standard cubic centimeters per minutes). The furnace temperature was adjusted to $900-1000{ }^{\circ} \mathrm{C}$ and heated up uniformly from room temperature. After 1-2 hours the system was cooled down. The resulting material was a white colored material around the inner walls of the quartz boat. The experiment was repeated for the n-type GaAs without gold and similar white colored wool shaped material was observed around the boat in addition to some long glass needle shaped structures sticking out of the boat. Also undopped GaAs (Aldrich, 99.99\%) with/without gold was tried as initial material, interestingly it didn't grow any structure, although the

same powder mixed with sulfur (with weight percent of (0.999: 0.001) has resulted in Gallium oxide synthesis (in chapter III we discuss more about the structures of the above 
growth conditions). In all the trial processes discussed above, the same temperature and gas flow condition have been applied.

\section{Design and set-up of the pulse laser vaporization system}

Pulse laser vaporization is a versatile method for nanowire, nanoparticles and nanostructures synthesis. The basic function and capability of PLV has been covered in "Chapter I". Now the design and details of the PLV system built in the lab will be described. Figure 23 shows the schematic of PLV has been designed and manufactured.

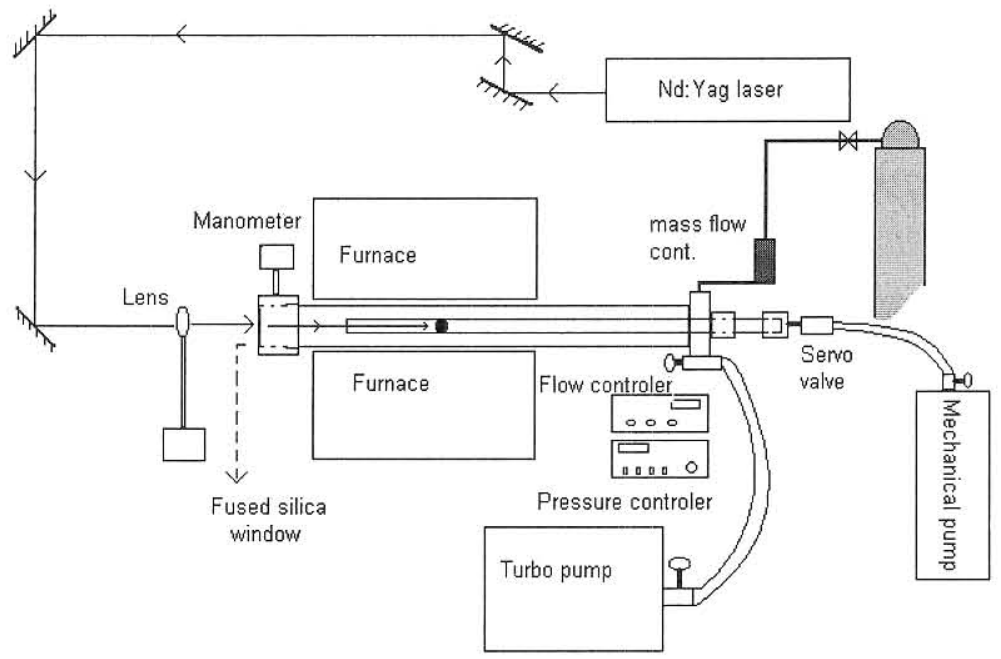

Fig.23 Schematic of Pulse laser vaporization system

The PLV has several important features and accessories as follow: high power pulse laser, optics, reactor, furnace, target holder, stepping motor, pressure controller and gas flow controller. The laser is a Q-switched Nd: Yag laser, which operates in pulse mode with two possible frequencies, $10 \mathrm{~Hz}$ and $20 \mathrm{~Hz}$. The output beam includes the first, second and third harmonic of the laser, wavelengths of $1064 \mathrm{~nm}, 532 \mathrm{~nm}$ and $355 \mathrm{~nm}$, separable with an optics setting (it is optional choice). Pulse width is 5-7 ns for the first 
harmonic and 4-6 ns for the other harmonics. Its power is 4.6 watts for $1064 \mathrm{~nm}, 2.1$ watts for $532 \mathrm{~nm}$ and 1.4 watts for $355 \mathrm{~nm}$. The laser beam is reflected from four mirrors (purchased from RMI) to direct the beam into the converging lens and focused into the reactor toward the target. The mirrors are reflective for wavelengths between $550 \mathrm{~nm}$ to $1200 \mathrm{~nm}$, and are transparent for the rest of the wavelengths. In Figure 24 the reflectivity versus wavelength of the mirrors is depicted. The reason for the use of four mirrors is to save space on the optical table, where PLV is mounted on.

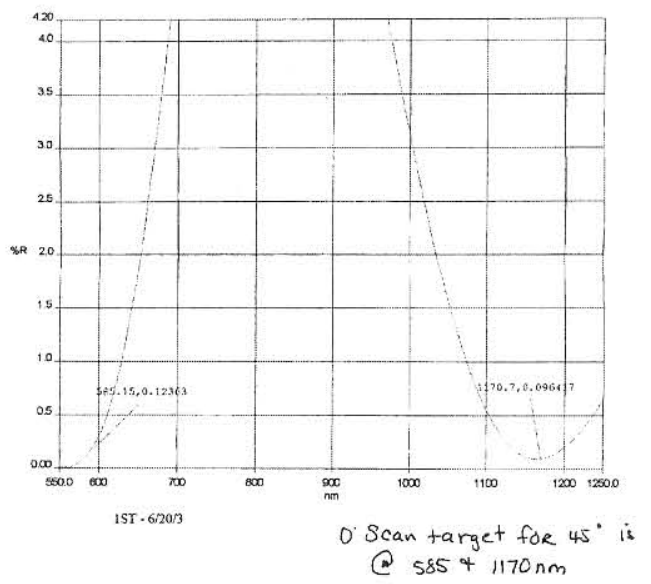

Fig. 24 Graph of reflectivity of mirrors vs. the wavelength

The reflected beam is directed through a converging lens (RMI, convex lens) with a focal length of $76 \mathrm{~cm}$ (for $\lambda=1064 \mathrm{~nm}$ ). The lens has a high transmission coefficient for the primary and second harmonic of the laser beam.

The reactor is a $3.8 \mathrm{~cm}$ quartz tube concentrically mounted inside a $5.08 \mathrm{~cm}$ quartz tube. The inner tube resides on two half circle poco graphite pieces, purchased (Pocostore) and machined carefully to make the tubes concentric. The outer tube sits in the hinged tube furnace and connected to a stainless steel head housing a "fused silica" window. This head has provisions to a stainless steel QF flange (Kurt J. Lesker) to support a 
capacitance manometer. The quartz tube is connected at the other side to a QF quick connector (Kurt. J. Lesker), which in turn connects to a QF cross with three other sides connected to gas inlet, turbo pump and to the outlet of the system (a servo-valve and a mechanical pump). This arrangement of the tubes allows the gas to uniformly heat up in the space between the outer and inner tubes before entering the inner tube, where the reaction takes place, this provides equally heated inert media and all the catalyst particles and reactant materials get heated to the same condition for more homogenous growth.

For controlling the vacuum the PLV apparatus is equipped with two pumps, a turbo pump (Edwards) is used for initial pumping of the reactor down to 0.5 torr, through a stainless steel valve. Then the turbo pump is isolated from the system by closing the angle valve and a mechanical pump is opened to the reactor through a manual valve (Kurt J. Lesker) and a servo-valve (or control valve, Edwards, model DM20). The pressure is controlled with the servo-valve, which opens the outlet according to the pressure setting at the pressure controller box (Edward, model 1501C) and the pressure monitored with a capacitance manometer (Edward, model 600 Barocel). In this configuration the servovalve is placed downstream on the system, which is a better way to control the pressure of the reactor while some gas is flowing and the pump is operating through the servovalve down stream. The servo-valve is a solenoid controlled compact device.

The fluidic part of the PLV system is a mass flow controller (MKS, model 1179A), which transport gas from the source through a small manual gas valve and the $1 / 4$ inch stainless steel tubing to one side of the QF cross that has been discussed before. The mass flow controller is connected to a controller (MKS, type 247), which can access four different channels and set the flow rate with an accuracy of $0.1 \mathrm{sccm}$. 
The furnace is a one-zone furnace (Barnstead International, model 97300), equipped with a programmable temperature controller. It can be programmed for three ramps, ramping to the first set point and dwell time and second ramping (which in our experiment is usually cooling down to room temperature).

The converging lens is mounted to a support on the top of a stepping motor (Velmex, Inc. Series MA2500) and (VXM) controller, and operated via computer with "Lab View". The reason for use of the stepping motor is to raster the laser beam on the target. Since the laser spot is very sharp after focusing with the converging lens $\left(0.5 \mathrm{~mm}^{2}\right)$, for easy penetration. In order to have more material ablated, it is better to scan the target horizontally and vertically.

The target holder is a poco graphite piece (Pocostore, 10381 AXM-5Q ground plate 0.63 $\mathrm{cm}$ ) machined according to the size of the target; it is made of two similar pieces with four legs to sit inside the inner tube reactor and screwed with molybdenum screws and nuts (molybdenum has a higher melting temperature than the PLV temperature).

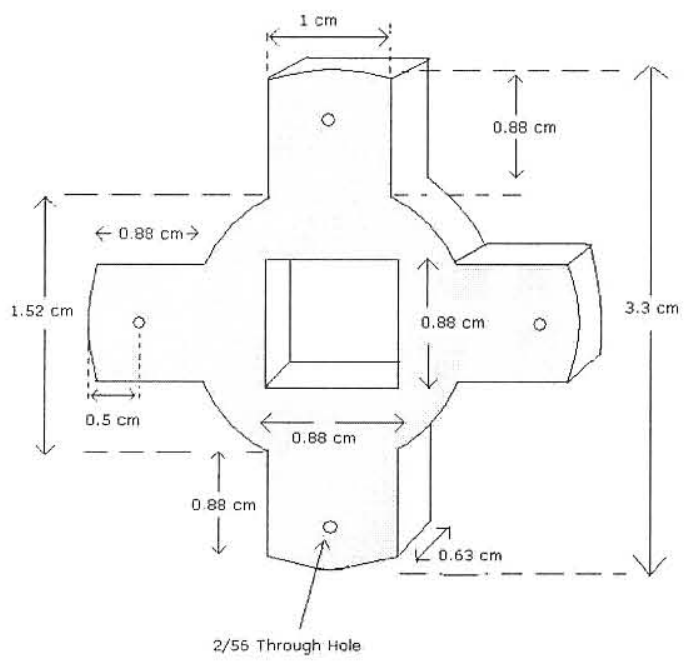

Fig.25 Schematic of graphite target holder 
The schematic of target holder is shown at Figure 25. The poco graphite is a high temperature material especially in inert media. The target is a mixture of reactant materials, sometimes mixed with catalyst, pressed in a stainless steel pellet maker with a diameter of $1.27 \mathrm{~cm}$ (Fig.26.b). It has two cartridge heaters to heat up the powders mixture, and a thermocouple to read the temperature. The heaters and the thermocouple are connected to a temperature controller (Eurothearm controls, model 808/847), which is programmable for set point, ramp rate, and dwell times for two segments.

In Figure 26 a picture of the PLV apparatus is shown and its accessories and details.

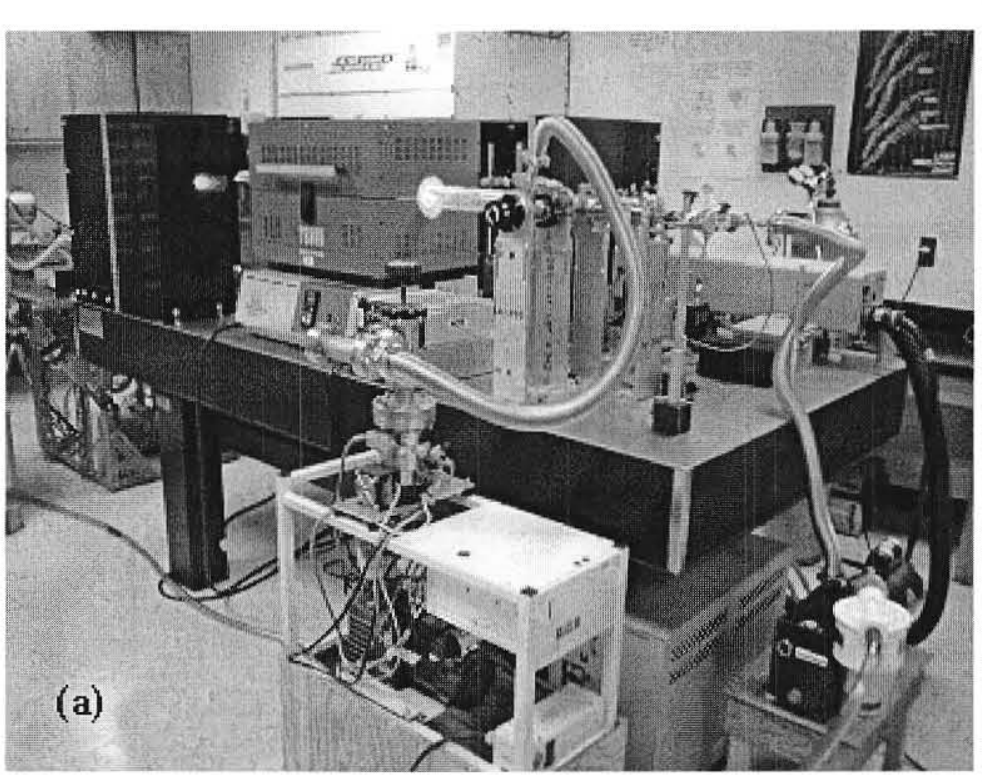

Fig.26 (a) PLV set up in the lab in the lab (b) pellet maker (c) target

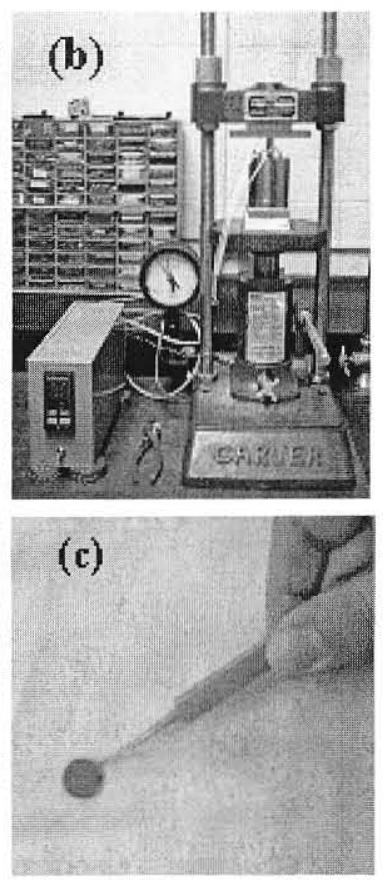

\section{PLV growth of some nanowires}

Several successful syntheses have been accomplished with PLV. The advantage of this method is that the growth procedure is very clean and the nanowires or nanotubes are relatively pure and long. For all growth procedures in our experiments the laser beam was 
maintained at $10 \mathrm{~Hz}$ frequency and consisted of the first and second harmonic $(\lambda=1064$ $\mathrm{nm}$ and $532 \mathrm{~nm})$.

GaAs nanowires were grown by PLV; GaAs powder (Aldrich, 99.99\%) was mixed with gold powder (Alfa Aesar, spherical, 0.8-1.5 micron) with atomic percentage of GaAs:Au (0.95:0.05), uniformly mixed, and hot pressed in the stainless steel pellet maker at a hydraulic presser of $6000 \mathrm{psi}$ and temperature of $200{ }^{\circ} \mathrm{C}$ for about five hours, then cooled to room temperature resulting a coin shape pellet (Fig.27 shows the figure of the target holder). Then the target was placed in the target holder inside the inner tube at the focal point of the lens, and the system evacuated down a pressure of at most 0.5 torr with the turbo pump. Then the valve was opened and flow was started, while controlling the flow by means of mass flow controller at flow rate of $100 \mathrm{sccm}$.

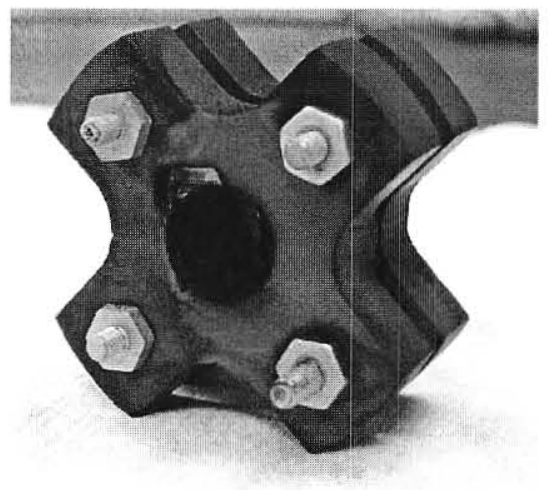

Fig.27 Poco graphite target holder target holder

The pressure controller was set up for 300 torr with the servo-valve in auto mode for automatic pressure control according to the set point (Notice that the second pump, mechanical pump, was pumping through the servo valve). While the inert gas was flowing, the furnace was slowly heated at a rate of $10{ }^{\circ} \mathrm{C} / \mathrm{min}$ high as $950{ }^{\circ} \mathrm{C}$, and maintained at that temperature for 4 hours and then cooled down to room temperature $\left(10^{\circ} \mathrm{C} / \mathrm{min}\right)$ 
When the furnace temperature reached $900-950{ }^{\circ} \mathrm{C}$, the laser and the stepping motor were turned on. The stepping motor used in the lab sweeps only in the $\mathrm{x}$-direction (horizontally); so the knob on the adjusting frame of the reflecting mirror was used to manually scan the beam in y-direction (vertically). The laser was turned off before the furnace started to cool. Essentially PLV produces plasma of GaAs and LAG and VLS growth methods result in nanowires/nanotubes synthesis. After the system was cooled down, gray- black ashes were observed around the inner tube outside of the furnace, where it was cool enough for nanowires to collect on. More information about the growth result will be presented in chapter III. It is important to accurately scan only the target area but not the graphite holder to avoid too much carbon residue on the collected materials.

GaP nanowires have been grown under similar conditions. GaP crystalline pieces (Aldrich, 99.99\%0 were ground and mixed with gold powders (Alfa Aesar, spherical 0.81.5 micron) with atomic percentage GaP:Au (0.95:0.05). Then the mixture of powders was loaded in the pellet maker and heated up to $200{ }^{\circ} \mathrm{C}$. The target was then attached to the target holder inside the reactor and the furnace was heated up to $850-950{ }^{\circ} \mathrm{C}$ at a ramp rate of $15{ }^{\circ} \mathrm{C}$ while the argon gas was flowing, and held at that temperature for 3 hours. The pressure was kept at 300 torr and the flow was $50 \mathrm{sccm}$. Material collected at the cold part of the inner tube had a light brown color. (More information in chapter III)

Silicon nanowires were synthesized using flat pieces of silicon, and gold/iron nanoparticles (catalyst), and deposited on a silicon wafer substrate. By immersing the $\mathrm{Si}$ substrate in iron solution (iron nitride, $\left.\mathrm{Fe}\left(\mathrm{No}_{3}\right)_{3}\right)$ ) and then washing with hexane the iron particles replaced with some atoms on the substrate. To obtain gold nanoparticles the 
substrate was immersed in gold colloidal solution for less than a minute. The substrates with iron/gold catalyst placed in other side of the target. Argon gas was applied as an inert gas flowing at a rate of $50 \mathrm{sccm}$, and the temperature of the furnace was increased to $1200{ }^{\circ} \mathrm{C}$. The laser ablation of the target lasted for 3 hours at this temperature. The nanowires were grown on the substrate from the catalyst particles. The growth result will be discussed more in chapter III. Also Silicon nanowires were grown using a pellet of $\mathrm{Si}$ mixed with $\mathrm{Au}$ micro particles. The pellet was a mixture of $(\mathrm{Si}: \mathrm{Au})$ with molecular ratio of (0.95:0.05), hot pressed at $200{ }^{\circ} \mathrm{C}$ for 4 hours under 5000 psi pressure. The PLV method was applied and the pressure kept at 300 torr while argon gas was flowing with rate of $100 \mathrm{sccm}$ at temperature of $1200{ }^{\circ} \mathrm{C}$ for one hour. The growth was successful and nanowires were collected on the wall of the PLV quartz tube at the cool part.

Another successful attempt was heterostructure GaAs/GaP nanowires. The target was half GaAs and half GaP fixed in the graphite holders. The temperature was kept at $850^{\circ} \mathrm{C}$. The catalysts particles used were iron/gold and the same method mentioned in last paragraph was used to disperse particles on the substrate. The stepping motor was programmed differently than in the previous case (Fig.28); the laser beam raster each half of the target for a short time ( $30 \mathrm{sec}$.) and then switched to the other half.

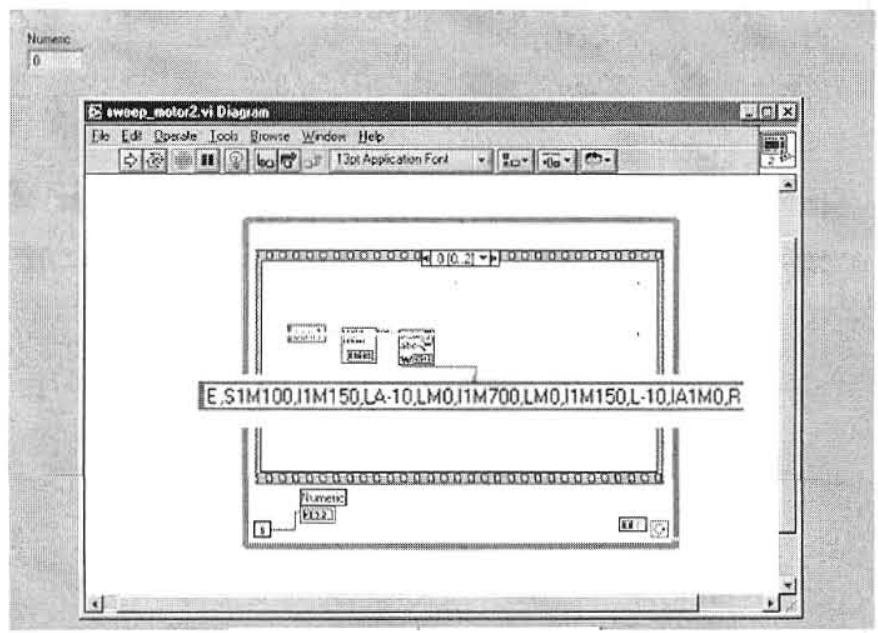

Fig.28 LabView screenshot for scanning the $\mathrm{GaP} / \mathrm{GaAs}$ target 
The growth was successful mostly from the substrate, which was at the side of the target. The flow rate was $80 \mathrm{sccm}$ and pressure was 300 torr. The scanning path of the laser is shown in Fig.29.

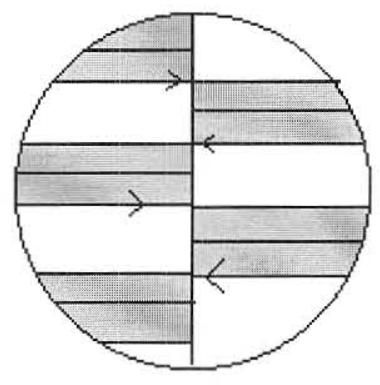

\section{Fig.29}

Scanning path of the laser on $\mathrm{GaP} / \mathrm{GaAs}$ target

SWNTs were synthesized using the PLV method. The target was made of a mixture of carbon, nickel and cobalt, as transition metal catalysts. The molecular ratio of the three elements was as follow, C: Ni: Co (91\%: 4.5\%: 4.5\%) and they were mixed thoroughly and hot pressed under $5000 \mathrm{psi}$ pressure at $200{ }^{\circ} \mathrm{C}$. The target was secured in the graphite target holder, placed in the PLV system, and heated up to $1200{ }^{\circ} \mathrm{C}$. The pressure was kept at 500 torr with argon gas $150 \mathrm{sccm}$ flow rate, and the ablation lasted for 5 hours. 


\section{CHAPTER III}

\section{CHARACTERIZATION}

\section{Results and discussion}

This chapter is mainly focused on the results of nanowire synthesis including structure analysis and chemical and physical properties. Several surface science techniques were employed to ascertain the identity, shape, crystal structure and chemical composition of the synthesized material. Analysis has been done on each material with more thorough study on certain materials and less analysis on others due to unavailability of some facilities in the period of time this thesis project progressed. SEM, AFM, TEM, XRD, EDS and Raman studies on some of the samples are presented in the following sections.

\section{$\mathrm{Ga}_{2} \mathrm{O}_{3}$ nanowires and herringbone structures}

Gallium oxide synthesis was one the most interesting and unexpected experimental results achieved. PVD growth of gallium oxide covered in the last chapter, and we observed Te doped gallium arsenide powder with or without Au results in growth of $\mathrm{Ga}_{2} \mathrm{O}_{3}$. The same result was observed for undoped $\mathrm{GaAs}$ powder mixed with $\mathrm{S}$ and it is expected that the same growth would happen for the mixture of $\mathrm{GaAs}$ and $\mathrm{Se}(\mathrm{S}, \mathrm{Te}, \mathrm{Se}$ belongs to group $\mathrm{V}$, challcogenide). A summary of $\mathrm{Ga}_{2} \mathrm{O}_{3}$ growth result is presented in table2. 
The final growth product was a white woolly substance covering the inner side of the boat. Some crystalline needles were pointing out of the edge of the boat. Some of the

\begin{tabular}{|l|c|c|c|}
\hline & $\mathrm{Au}$ & $\mathrm{S}$ & Nothing \\
\hline GaAs /Te (doped) & Yes & NR & Yes \\
\hline GaAs (undoped) & No & Yes & No \\
\hline
\end{tabular}

Table 2

structures were larger enough to be visible optical microscope. To see smaller structures with more details, scanning electron microscope "LEO 1430" was used. The images showed the same morphology as the big structures. Fig.30 ( $a, b$ and $c$ ) shows the images of the results for the three experimental approaches: GaAs doped with Te, undopped GaAs mixed with $\mathrm{S}$ and $\mathrm{GaAs}$ doped with $\mathrm{Au}$. The growth structure slightly changed for each synthesis. However the resulting material was beta-gallium oxide for all three experiments. Presence of tellurium (a challcogenide) in gallium arsenide results in nanowires and herringbone structures. GaAs/Te mixed with gold resulted in nanowires
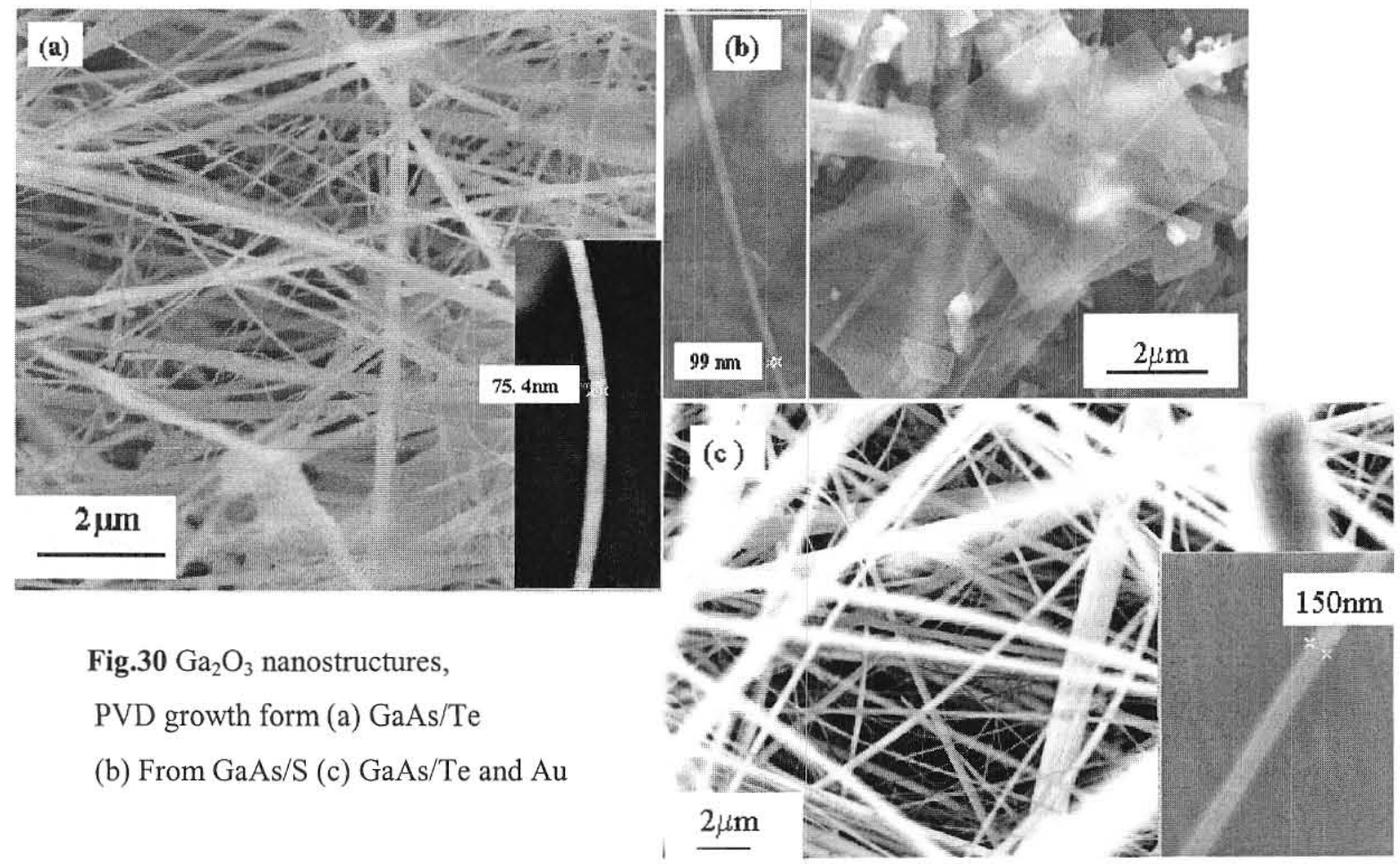

Fig. $30 \mathrm{Ga}_{2} \mathrm{O}_{3}$ nanostructures, PVD growth form (a) GaAs/Te

(b) From GaAs/S (c) GaAs/Te and $\mathrm{Au}$ 
with smaller diameter. The nanowires produced from GaAs/Te looked like ribbons and wires from $\mathrm{GaAs} / \mathrm{Te}$ and Au have a cylindrical cross section more like rods.

$\mathrm{GaAs} / \mathrm{S}$ produced nanowires and herringbone structures and most of the nanowires looks like sheets and ribbons. The structures were grouped as ribbon structures with sharp edges, cylindrical needle structures with sharp tips, sheet-like structures with right angles, and herringbone structures with steps. The ribbons and needles resulting from GaAs/Te mixed with $\mathrm{Au}$ result in the smallest structures, ranging between 10-200 $\mathrm{nm}$ in diameter. The sheets and rods resulting from GaAs/S had bigger structures to about 50-500 nm. The width of the herringbone structures was found to range from 20 to10 $\mu$ m. Fig.31.a, b and $\mathrm{c}$ shows the ribbons, sheet and herringbone structures of gallium oxide.
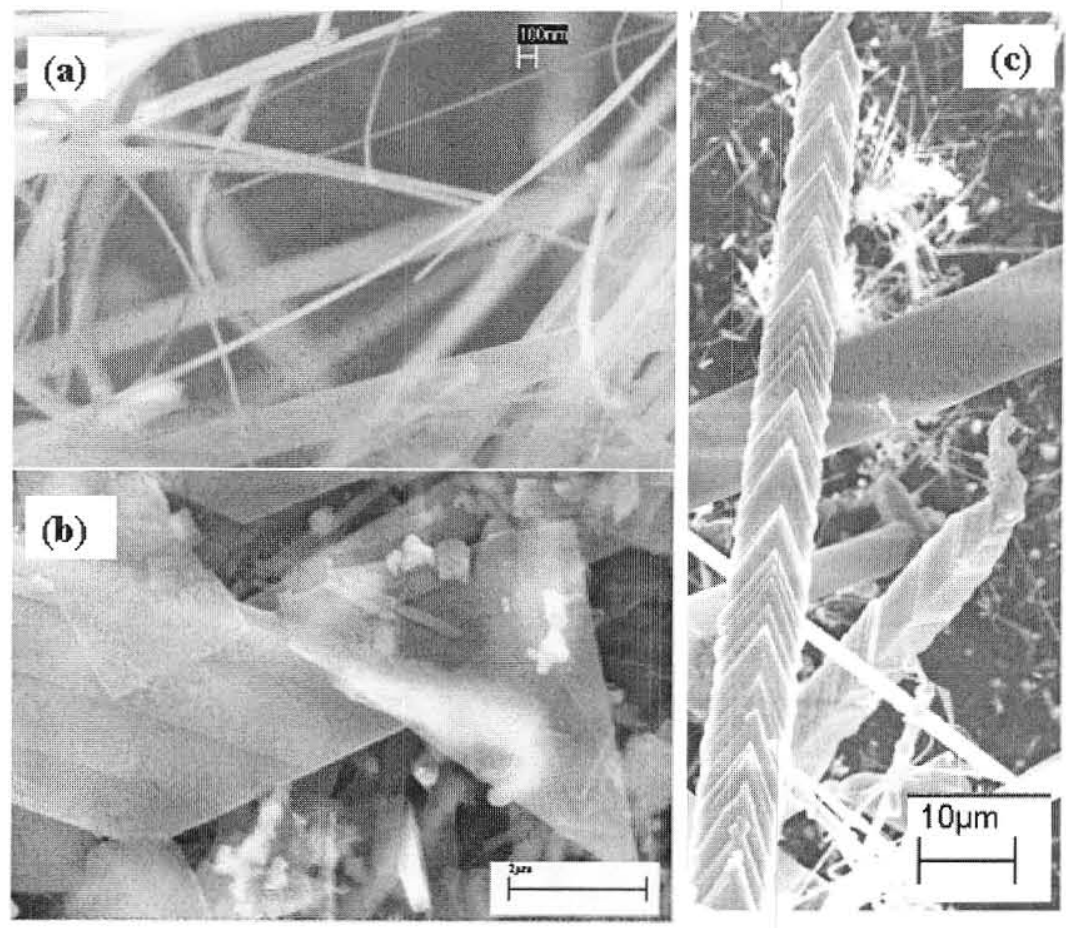

Fig.31 Ga2O3 (a) ribbons, (b) sheets and (c) herringbone structures 
The growth mechanism was assumed to be physical evaporation of Te doped GaAs powder. Ga get oxide with the oxygen in the reactor after decomposition, and arsenide leaves the reactor, and $\mathrm{Te}$ and $\mathrm{S}$ promote the reaction.

Fig.32.b shows the selective area diffraction ( $\mathrm{SAD}$ ) of nanowires (ribbons and sheets). The diffraction pattern reveals a single crystalline structure for $\mathrm{Ga}_{2} \mathrm{O}_{3}$. The high resolution TEM (HRTEM) image of nanowires, Fig.32.a, shows that the structure has a rectangular shape with $50 \mathrm{~nm}$ width and very small thickness.

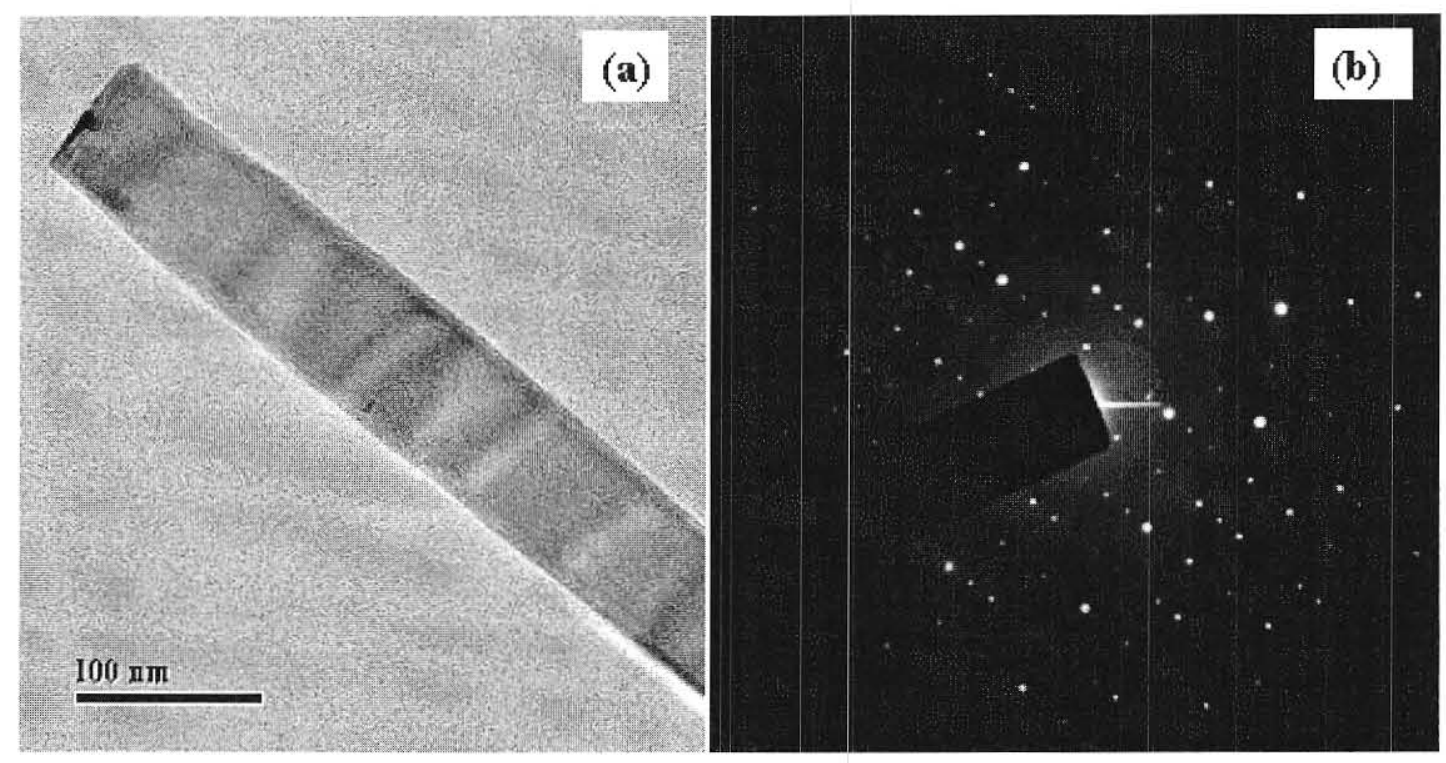

Fig.32 $\mathrm{Ga}_{2} \mathrm{O}$ nanoribbons (a) HRTEM image (b) SAD pattern

Fig.33.a shows a HRTEM image of the herringbone structure of gallium oxide and it exhibits some hexagonal crystalline facets located in the middle of the structure, with similar sequential spacing. The rest of the nanostrucure was a single crystal, and the crystalline planes were grown parallel to two edges of the hexagon facet. In the HRTEM images, the hexagons look darker than the rest of the structure which indicates for different crystallinty of them. Measurement of the interplanar spacing of the herringbone 
structure (the bright and dark regions of structure, shown in Fig.33.b) was done and the spacing for the hexagonal region was found to be about $0.58 \AA$ and for the rest of the structure about $0.29 \AA$.

The SAD pattern, Fig.33.a, shows the crystallinty of the herringbone structure. In this pattern two of the closest neighbors to the central spot were indexed, ( $\overline{2} 02)$ and (002). Using these two planes and the measured lattice spacing from HRTEM image the growth
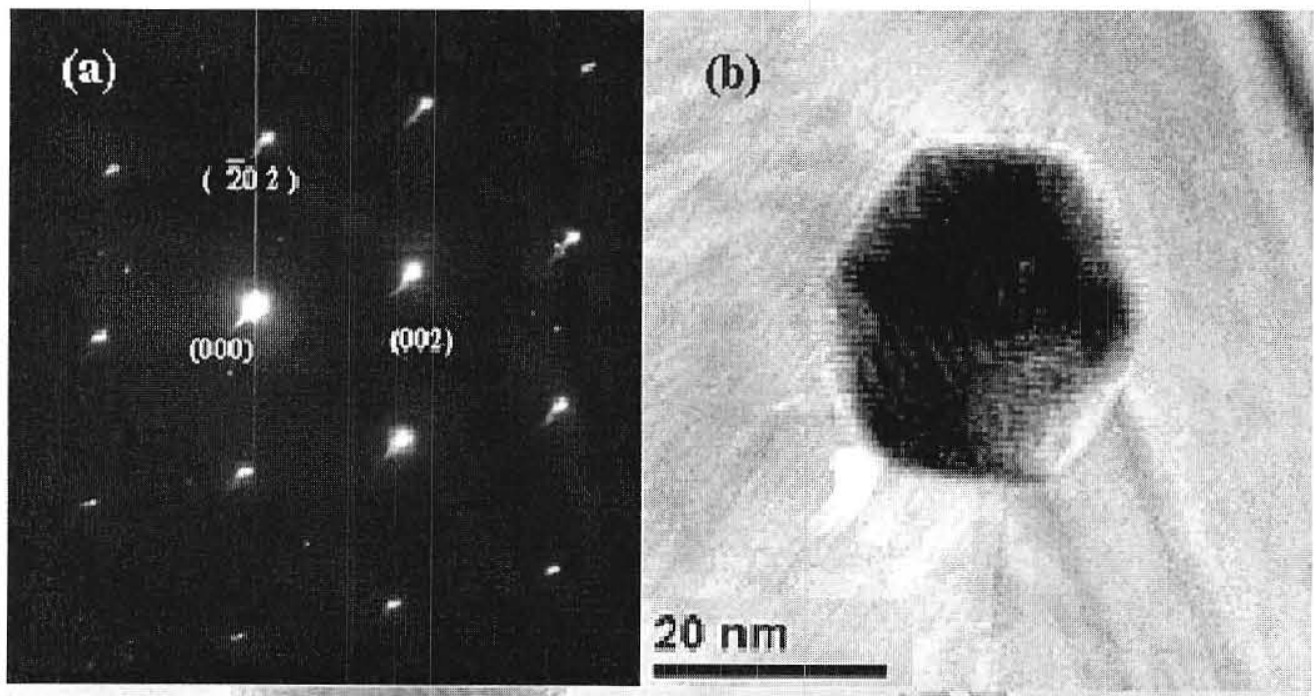

(c)
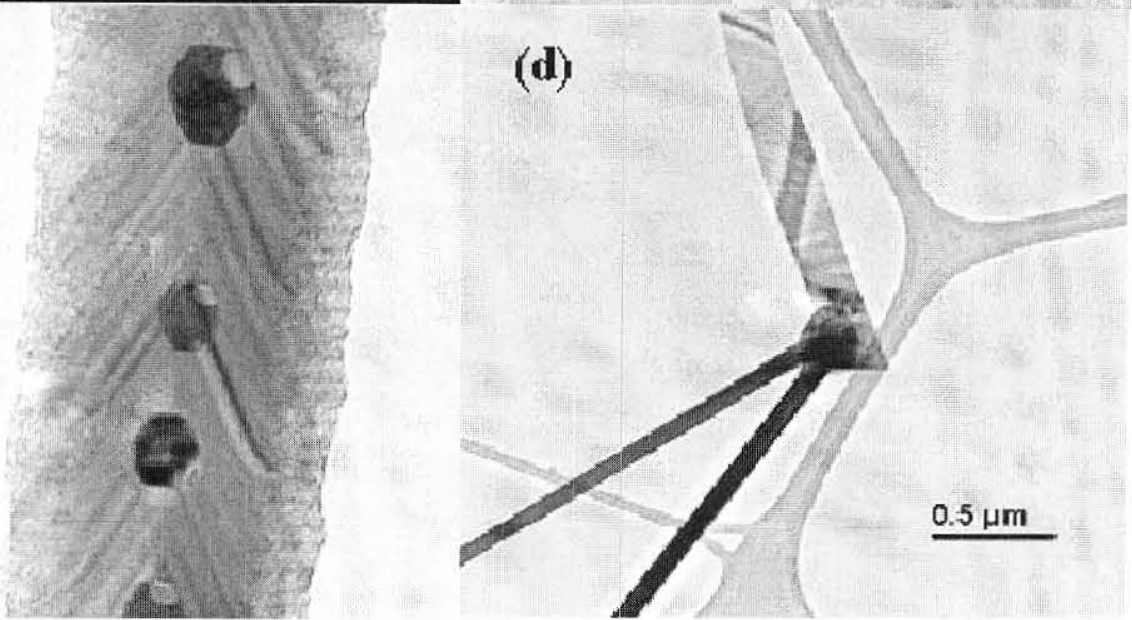

Fig.33 (a) SAD diffraction pattern of nanostrucure (b) TEM image of herringbone structure (c) another HRTEM image (consider the distances of hexagons) (d) ribbons HRETM images and it shows that the ribbon is not flatten on the surface 
direction was determined. The growth direction for herringbone structure made $35^{\circ}$ with [002] direction. For this diffraction pattern the zone axis assumed to be along [010].

The electron beam spot size of the TEM was about $80 \mathrm{~nm}$, so it can be seen that the diffraction resulted from both crystalline structures According to calculation, the diffraction pattern from herringbone structure didn't show the diffracted spots from the hexagon facets. It is because hexagons are a small part of the herringbone structure.

There are some debates about the crystal growth of these structures. It has been said that tellurium didn't participate in the growth, and it was not a VLS growth; but the presence of Te is essential as seen in the experiment. Arsenide exhausted from the reaction. A critical amount of oxygen was necessary in order to form gallium oxide. The nano-sized gallium oxide structures were initiated and the growth continued depending on the condition of the growth. Lattice mismatch and disorder in nanocrystals resulted in different final structures like nanowires (nanoribbons, nanorods and nanosheets) or herringbone structures. Hexagon facets behave like the initial seeds for the growth of herringbone structures and the lattice mismatches result in growth of crystalline parallel planes and this get repeated by new seeds, as in a nucleation process.

The XRD spectrum, Fig.34, was achieved by using a "Rigaku" diffractometer with a copper-K $\alpha$ x-ray tube, $\lambda=1.54 \AA$. The sample was a bunch of nanostructures scraped off of the white layer deposited on the quartz boat, and taped to a frame, which was placed vertically in the goniometer of the XRD system. The peaks of the spectrum identified the nanostructures as beta-gallium oxide. Beta Gallium oxide is a monoclinic crystal, with space-group $\mathrm{C} 2 / \mathrm{m}$ and lattice parameters, $\mathrm{a}=1.2214 \mathrm{~nm}, \mathrm{~b}=0.30371 \mathrm{~nm}, \mathrm{c}=0.57981 \mathrm{~nm}$, 
$\beta=103.7^{\circ}, \alpha=\gamma=90^{\circ}$. Each diffraction angle of the XRD spectrum belongs to a specific lattice spacing and each lattice spacing, $d$, represents a lattice plane.

The XRD spreadsheet data was plotted in "Igor-Pro”. Using Bragg's law (Eq.1), $d$ 9the lattice spacing) was calculated for corresponding $\theta$. Allowed lattice index regarding to each $d$ for a beta- $\mathrm{Ga}_{2} \mathrm{O}_{3}$ was checked from EMS on line [26]. Finally all peaks of XRD spectrum were indexed.

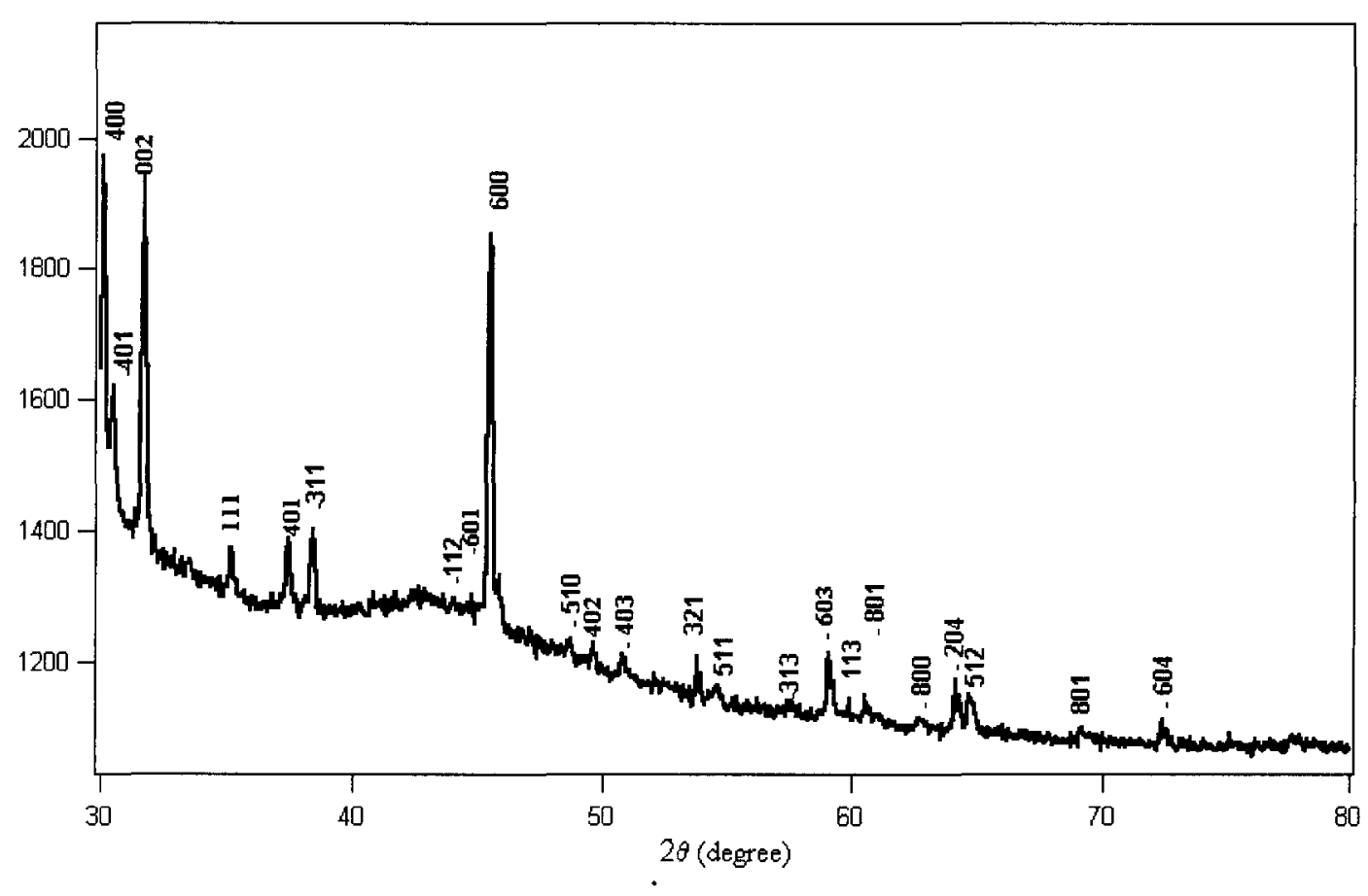

Fig.34 X-ray powder diffraction of the $\mathrm{Ga}_{2} \mathrm{O}_{3}$ nanostructures

Energy dispersive x-ray spectroscopy (EDS) has been used to investigated the $\mathrm{Ga}_{2} \mathrm{O}_{3}$ nanostructures, and the resulting spectrum showed that they were composed of $\mathrm{Ga}, \mathrm{O}$ and $\mathrm{C}$; No trace of As, Te, S or Au has been observed for three different approaches. The atomic ratio of $\mathrm{Ga} / \mathrm{O}$ was $2 / 3$ for most of the measurement. The spectrum of Fig. 35 
exhibits peaks of oxygen at $0.5 \mathrm{KeV}$, gallium at $1.1,9.3$ and $10.2 \mathrm{KeV}$, and finally carbon at $0.3 \mathrm{KeV}$.

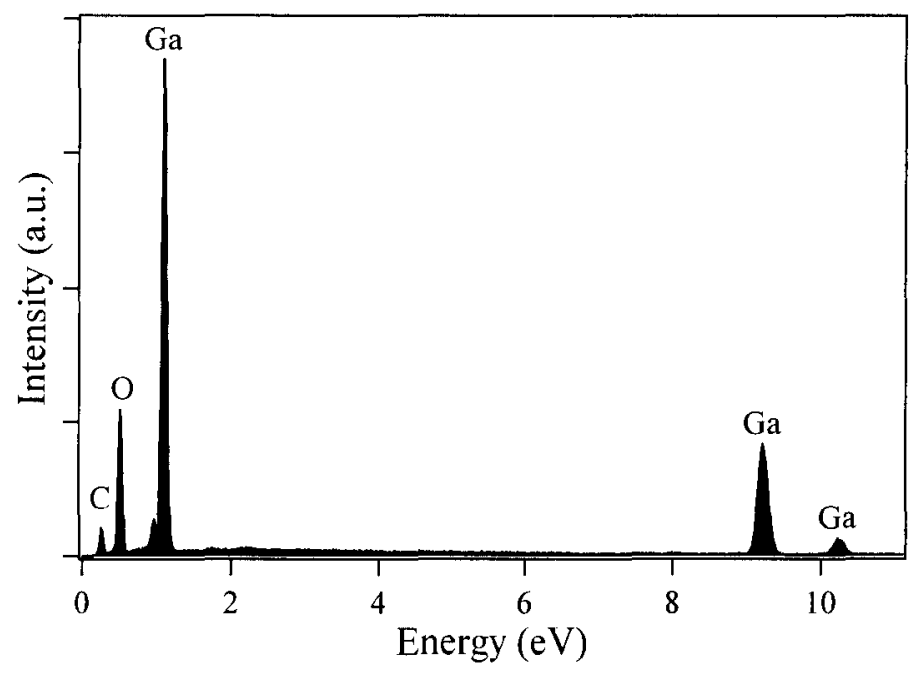

Fig.35 EDS

spectrum of

Gallium oxide

nanostructures

The EDS spectrum was acquired in the SEM system with an electron beam diameter of $1 \mu \mathrm{m}$, which meant that the detection spot included a number of ribbons and the resolution of the instrument was limited to bigger structures. However the EDS done with the TEM system has higher resolution, using a smaller electron beam spot size $(80 \mathrm{~nm})$. It shows the same composition of the nanostructure, $\mathrm{Ga}, \mathrm{O}$ and some $\mathrm{C}$. Some $\mathrm{Cu}$ was detected corresponding to TEM copper grid. (see Fig.36)

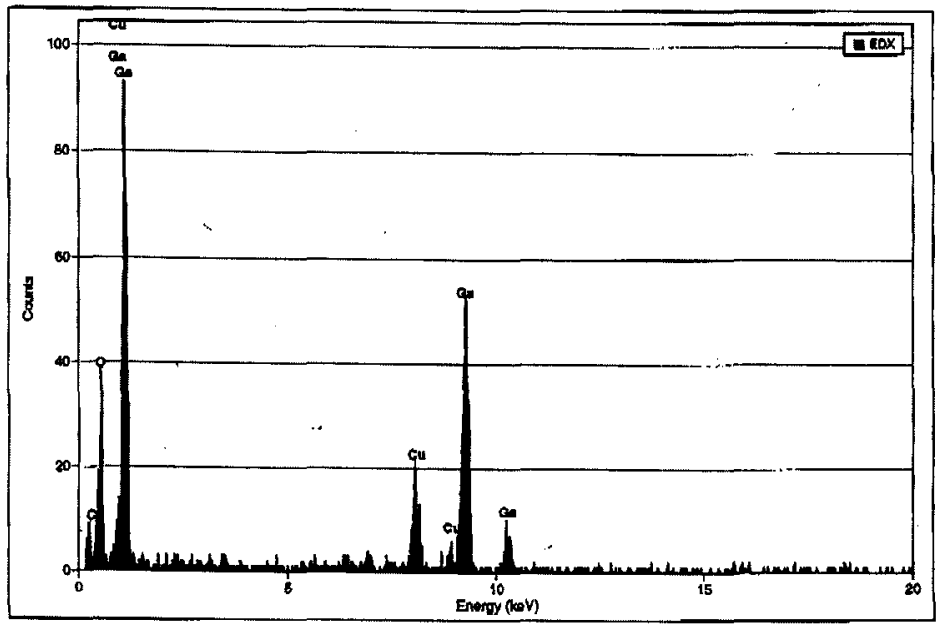

Fig.36 EDS spectrum of $\mathrm{Ga}_{2} \mathrm{O}_{3}$ smaller nanostructures taken with TEM

9000del.eml - Tha Jun 15 00:42:18 2004 
Raman spectroscopy of the gallium oxide nanostructures, Fig.37, was investigated and the result was identical with the spectrum of known gallium oxide nanostructures. Most of the peaks narrowed compared to the bulk gallium oxide Raman spectrum [23].

A Renishaw Micro-Raman "T64000" was applied to obtain the spectrum. The laser has $514.5 \mathrm{~nm}$ wavelength, $3 \mathrm{~mW}$ power, the exposure time of 900 seconds for 2 accumulations of spectrum, slit width $200 \mu \mathrm{m}$ and $3 \mathrm{~cm}^{-1}$ resolution of the CCD detector. The same features have been observed in the spectrum attained with a $488 \mathrm{~nm}$ excitation laser (2 mW), macro Raman "HR460" spectrometer. Both data were identical with known $\beta-\mathrm{Ga}_{2} \mathrm{O}_{3}$ Raman spectrums. This means the structure is high-quality gallium oxide nanostructures.

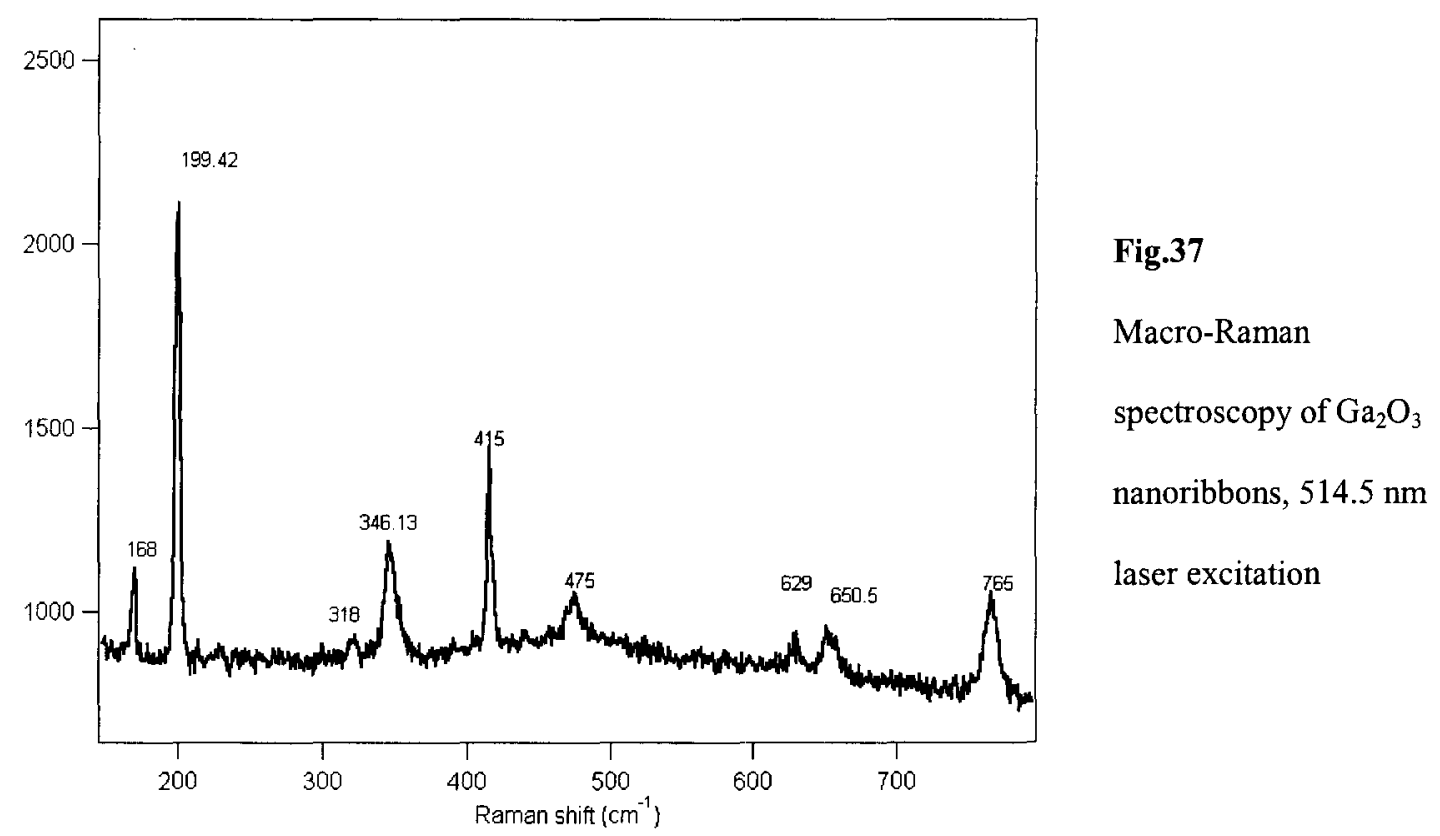

The AFM image, Fig.38, of the Gallium oxide nanoribbons dispersed on a substrate shows straight and rectangular structures of some ribbons. The width of the ribbons 
varies between $20 \mathrm{~nm}$ and $2 \mu \mathrm{m}$, in length between $1 \mu \mathrm{m}$ and $50 \mu \mathrm{m}$, and thickness of $5 \mathrm{~nm}$ to $50 \mathrm{~nm}$.

Fig. 38 AFM image of $\mathrm{Ga}_{2} \mathrm{O}_{3}$ nanoribbons. (the scale for vertical and horizontal axis is $\mathrm{nm}$ )

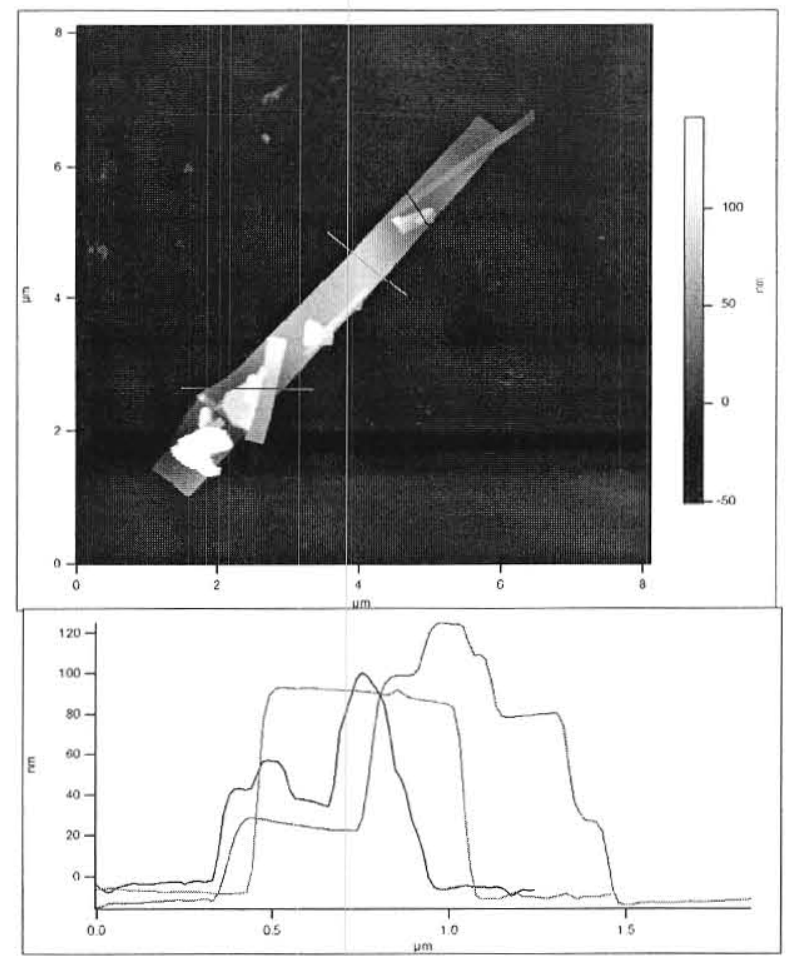

\section{Gallium Arsenide nanowires}

Gallium Arsenide nanowires are very important binary compound semiconductor materials with many potential applications in electronics and optics. GaAs synthesis was done applying laser assisted catalyst growth (LCG) method. Gold was used as catalyst for growth and after laser ablation it changed to a cluster of nanosize gold catalytic particles. The laser ablation provides plasma of reactant material while the furnace is at the set point. At this point the VLS growth of GaAs begins. 
SEM images of GaAs nanowires, Fig.39, indicated wire-shaped structures of final product on a silicon substrate. This substrate had been placed in the inner tube of the PLV system for easier collection of synthesis product. The diameter of the wires ranged between $20 \mathrm{~nm}-80 \mathrm{~nm}$ and length varies from $1 \mu \mathrm{m}$ to $10 \mu \mathrm{m}$. Most of the wires had straight structures with smooth surfaces, but some of the wires have curly structure. In both cases the catalyst was attached at the tip of the nanowires.

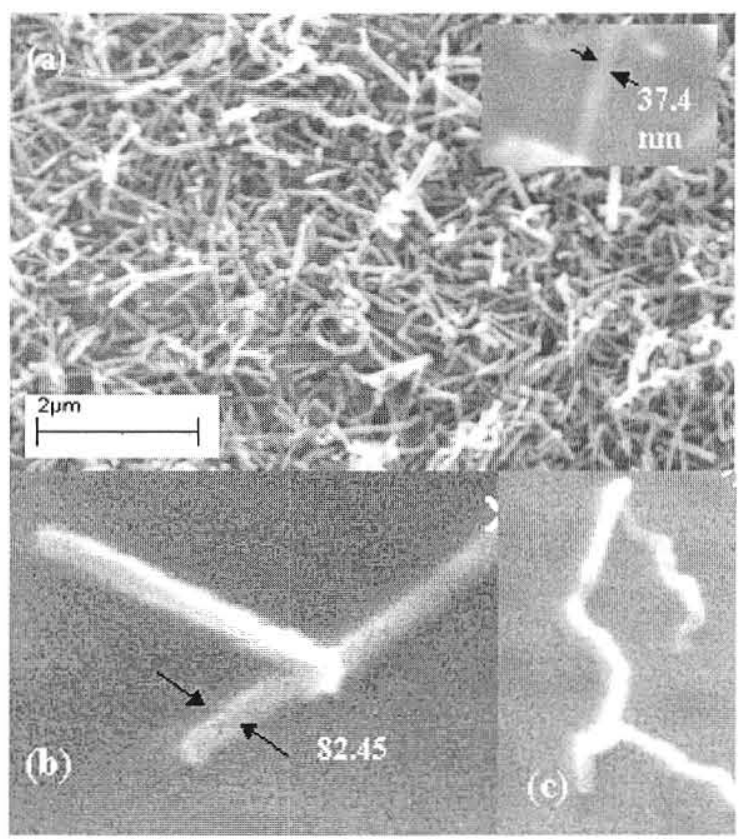

Fig.39 (a) GaAs collected on the silicon substrate (b) GaAs dispersed on the silicon; straight shape (c) dispersed curly nanowires of $\mathrm{GaAs}$

EDS data determined, Fig.40, the composition of nanowires to be $\mathrm{Ga}$ and As (equal atomic ratio) with some carbon and oxygen as impurities. Because of the limit of the resolution of EDS system, the gold wasn't detected and energy Dispersive x-ray spectroscopy of the nanowires showed mainly Ga and As. 


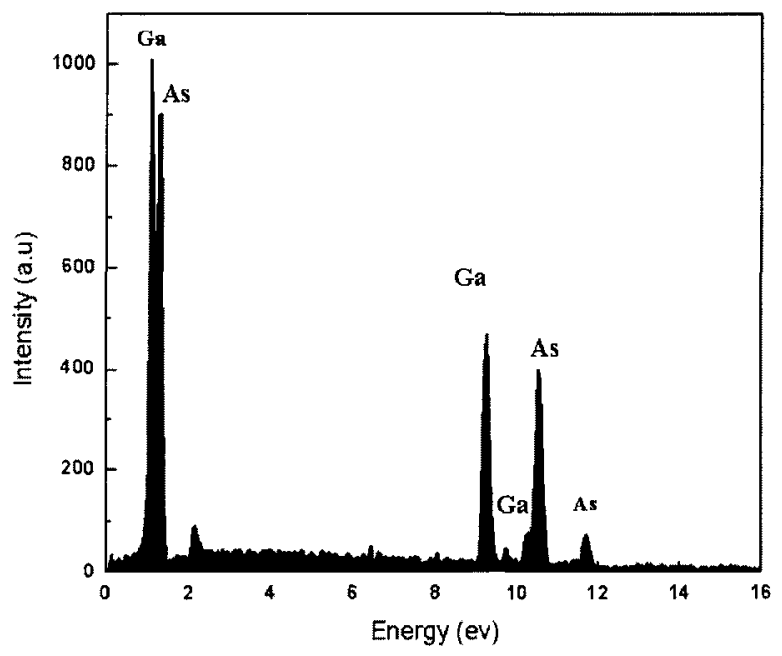

Fig.40 EDS of GaAs

nanowires

\section{Gallium phosphate nanowires}

The synthesis process explained in the previous chapter resulted in GaP nanostructure. SEM images, Fig.41, have shown nanowires of GaP. The structures looked thicker than GaAs; the reason might be because of the initial source of $\mathrm{GaP}$, which was ground powder of gallium phosphate crystalline pieces. As the initial powder was ground for longer time the growth product was straighter, longer and directional, otherwise they became thicker with a curled surface [24]. The diameter of the nanowires varied between $80 \mathrm{~nm}-300 \mathrm{~nm}$ and length between $5 \mu \mathrm{m}-20 \mu \mathrm{m}$. The growth product collected on the tube's wall. SEM images show that GaP nanowires grow out of some bigger clusters of gold and some of them grow directionally out of the clusters. 


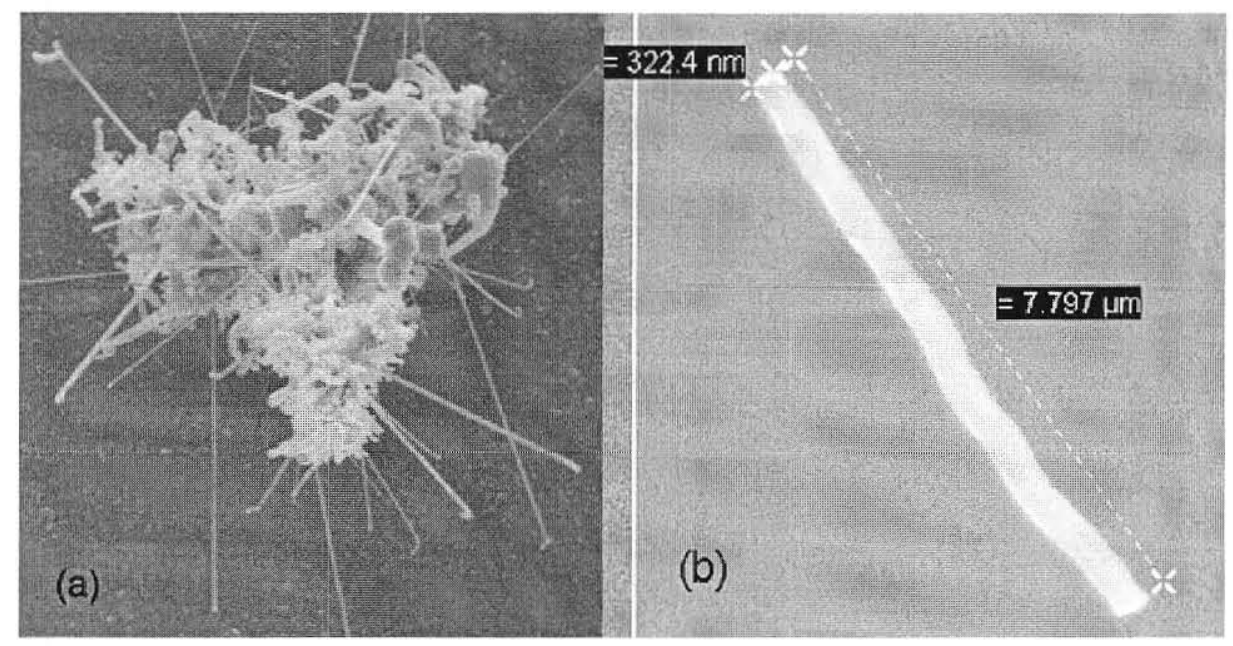

Fig.41 SEM images of GaP nanowires (a) deposited on a piece of silicon (b) dispersed on silicon

The EDS data, Fig.42, verified that the nanowires are composed of $\mathrm{Ga}$ and $\mathrm{P}$. The wires were big enough to be characterized by EDS system. After dispersing a solution of nanowires on $\mathrm{Si}$, the sample was taken to the SEM system and one wire was selected and EDS spectroscopy and mapping were done. The EDS spectrum shows the presence of $\mathrm{Si}$, $\mathrm{O}$, and even some $\mathrm{Al}$ resulting of the precursor stage.

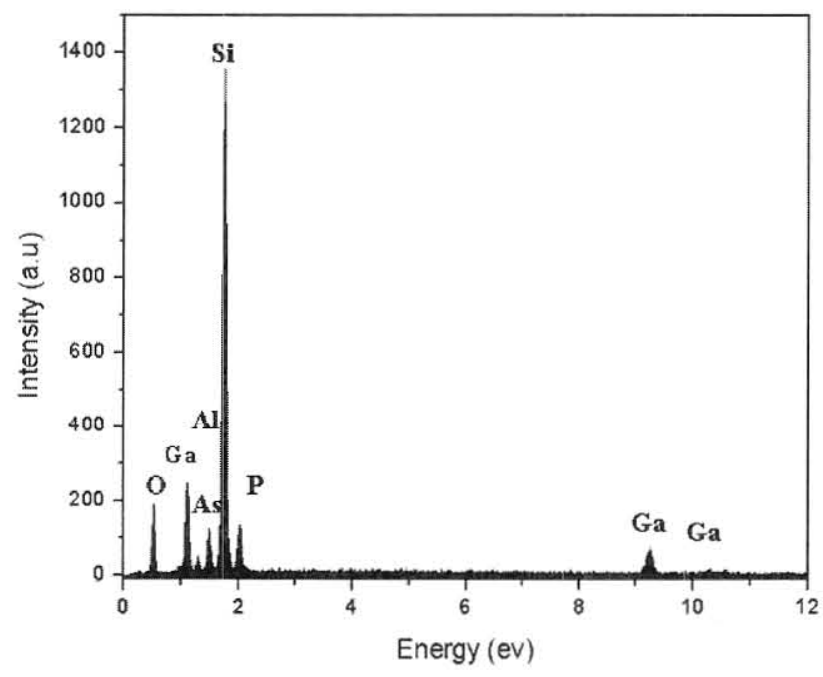

Fig.42 EDS spectrum of $\mathrm{GaP}$ nanowires 
The EDS mapping shows that the nanowires are pure $\mathrm{Ga}$ and $\mathrm{P}$ and the substrate is Silicon as depicted at Fig.43.

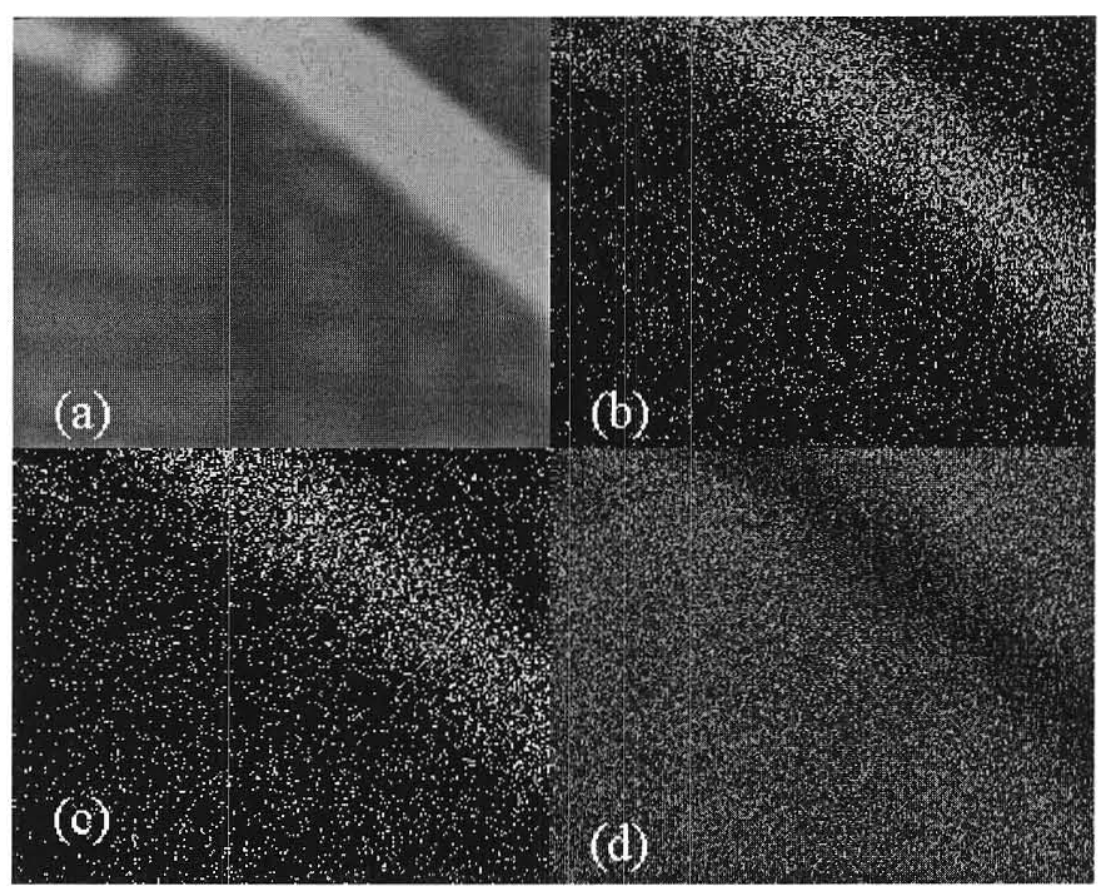

Fig.43 (a) The SEM image of GAP NW and EDS mapping of (b) Ga (c) P and (d) Si substrate with nanowires on the surface

\section{GaP-GaAs nanowires}

Attempts have been made to synthesize super-lattice nanowires composed of crystalline GaAs and $\mathrm{GaP}$. The SEM images indicated that the material collected on the tube's wall was nanowires. The exact growth mechanism is ambiguous, since the catalyst particles were deposited on silicon substrate before synthesis and it was expected the growth would happen mainly on the substrate (LCG growth mechanism). But the majority of the collected nanowires were on the wall of the inner tube and fewr wires were found on the substrate (as shown in Fig.44.a and b); Thus explanation of the growth mechanism of the 
wires collected on the tube's wall could be as follow; The floating catalytic particles, which separated from the substrates transported away with argon gas flow, and through VLS growth nanowires synthesized and collected on the tube's wall; or it could be an oxygen assisted growth, OAG, mechanism caused by minute amounts of oxygen leaked into the system. The synthesis succeeded for both types of catalyst particles, iron and gold; but iron catalyst seemed more promising for high yield growth on the substrate. Fig. 44 shows different structures from the three growth processes.)

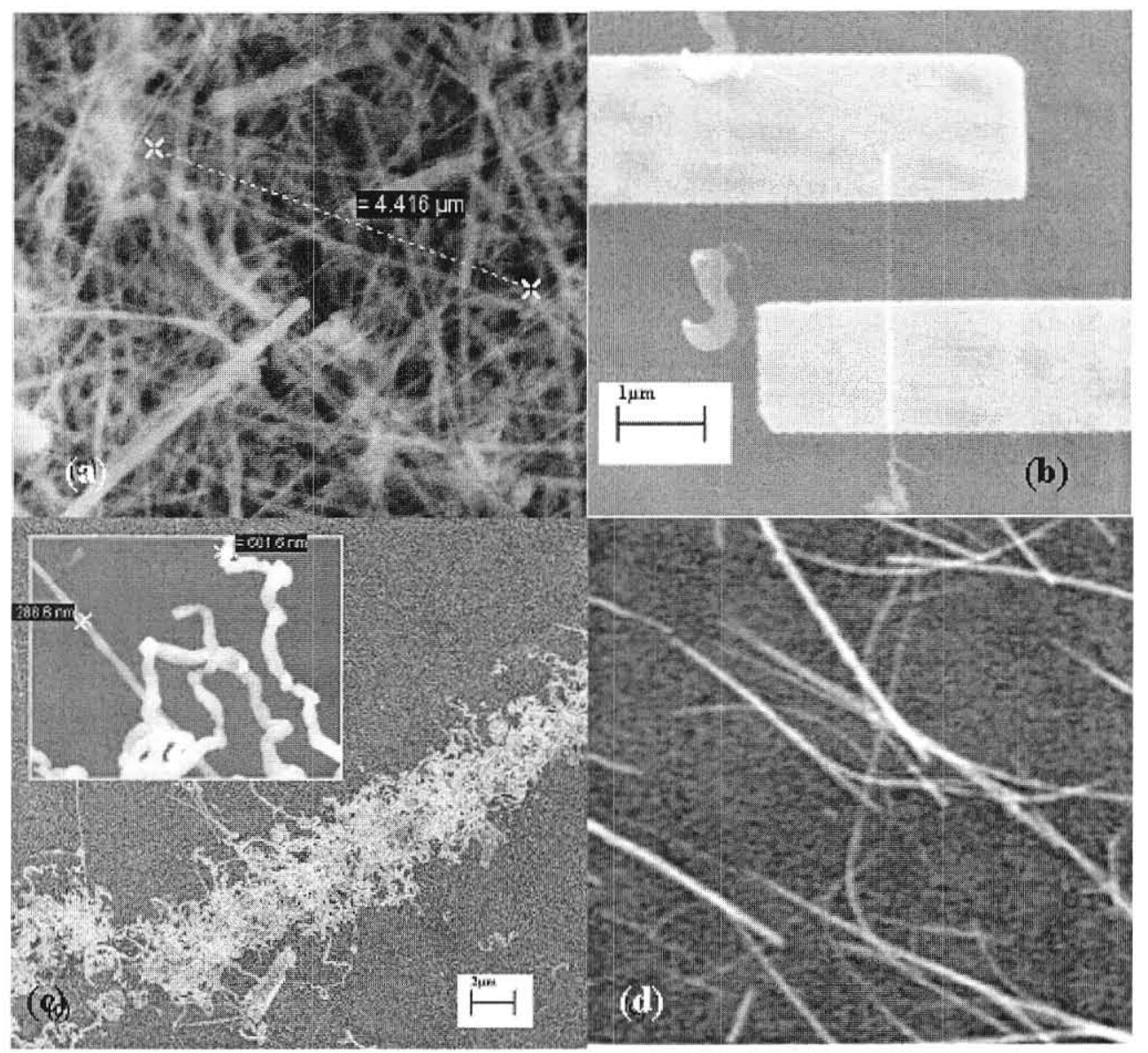

Fig.44 SEM images of GaAs-GAP hetero-structures (a) collected on the walls (b) Dispersed $\mathrm{GaP} / \mathrm{GaAs}$ NW on Si substrate with gold path (c) Fe as catalyst (d) Au catalyst 
The nanowires grown on substrate are larger with diameter $\sim 100 \mathrm{~nm}$ and length $\sim 15 \mu \mathrm{m}$. The collected materials on the tube's wall have smaller structures with diameter $\sim 30 \mathrm{~nm}$ and length $\sim 5 \mu \mathrm{m}$. (It was hard to image clearly these small nanowires with the limited SEM resolution.

Energy depressive x-ray spectroscopy of GaP-GaAs nanowires, Fig.45, revealed the presence of $\mathrm{Ga}$, As and $\mathrm{P}$ as constituent elements for NWs and some $\mathrm{C}$ and $\mathrm{O}$ as impurities. The EDS data on the materials collected from tube's wall indicates more oxygen identifying the growth be OAG. It is possible to extract information about the growth mechanism, using advance TEM and EDS on a very small area of the nanostructure, but lack of time and facility made it not possible to be completed during this project).

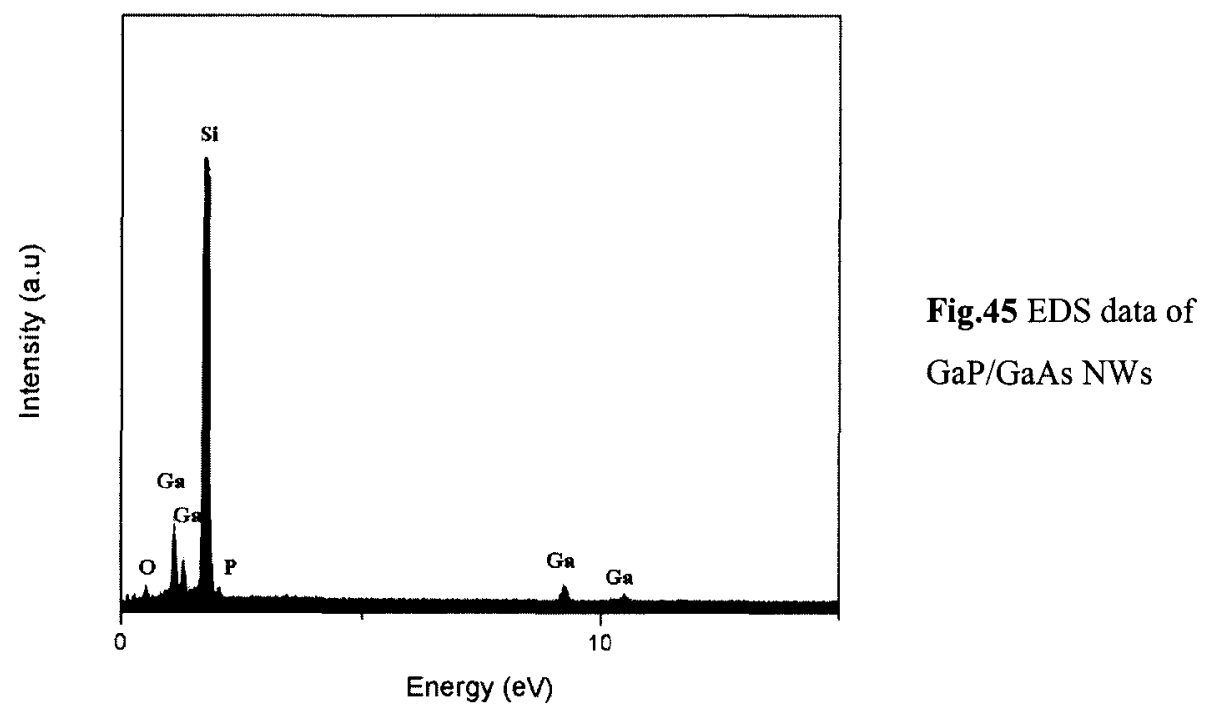

Fig.46 shows the Raman spectrum of GaAs/GaP structures taken by HR460 spectrometer with $488 \mathrm{~nm}$ laser excitation. The intense peak $\sim 266 \mathrm{~cm}^{-1}$ corresponds to GaAs like peak 
with a downshift. The broadened peak $\sim 355 \mathrm{~cm}^{-1}$ corresponds to a GaP-like peak. The shoulder peak $\sim 279 \mathrm{~cm}^{-1}$ could be due to the compound of $\mathrm{GaAs} / \mathrm{GaP}$.

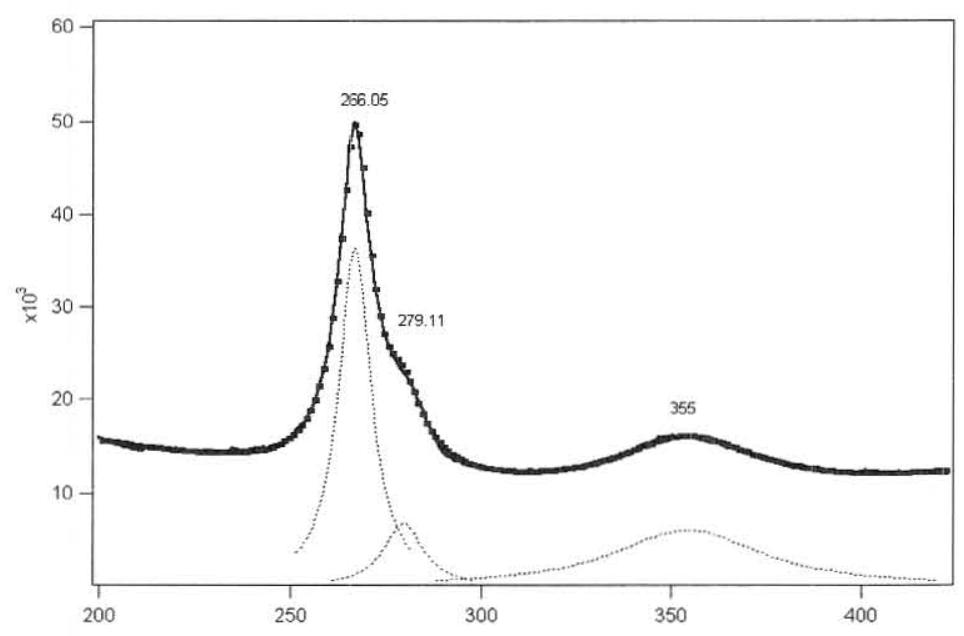

Fig.46

Macro Raman spectrum of GaAs-GaP NWs.

As shown in Fig.44.b, a nanowire has been dispersed on Si substrate and gold contacts were placed at both ends of it (using electron beam lithography and microfabrication), then IV measurement practiced in a probe station applying a DC voltage.

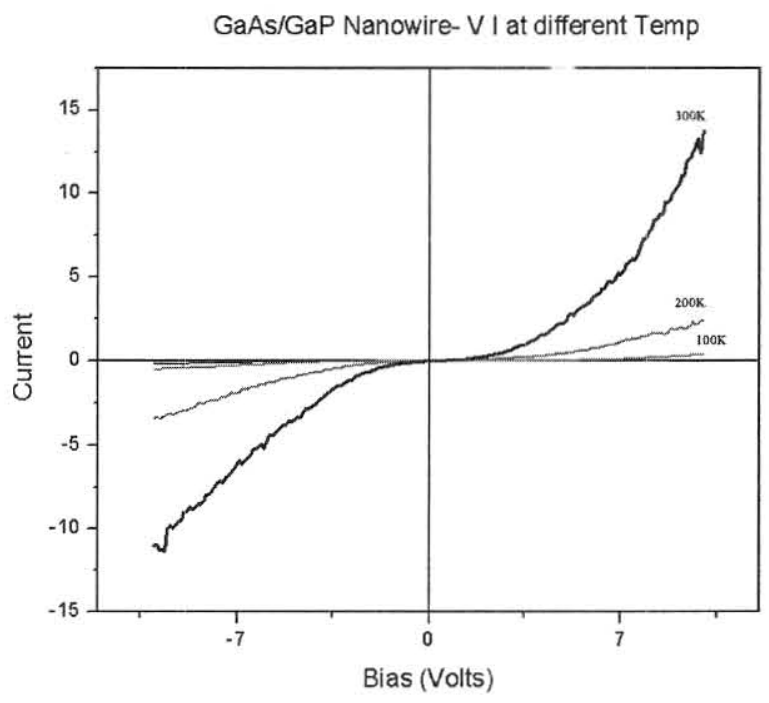

Fig.47. IV measurement of $\mathrm{GaP} / \mathrm{GaAs}$ nanowires 
The result of the measurement is shown at Fig.47. The sample displays a nonlinear, rectifying behavior, and strong temperature dependence.

\section{Silicon nanowires}

The experimental details of the synthesis of Si nanowires were explained in last chapter. The SEM images, Fig.48, are indicative of VLS growth with $\mathrm{Fe}$ or $\mathrm{Au}$ as catalyst particles. The growth essentially has been observed on the substrate and Ga did not act as catalyst. The Si nanowires grown with Au catalyst have diameters in the range of $20 \mathrm{~nm}$ $100 \mathrm{~nm}$ and lengths in the range of $5 \mu \mathrm{m}-20 \mu \mathrm{m}$. Si nanowires grown with $\mathrm{Fe}$ catalyst have larger diameters about $100-500 \mathrm{~nm}$ and lengths about $20 \mu \mathrm{m}-200 \mu \mathrm{m}$. However with a closer look it shows that larger clusters of catalyst nanoparticles resulted in uncontrolled growth at some locations of the substrate. This has to be optimized in the future by uniform distribution of catalysts.
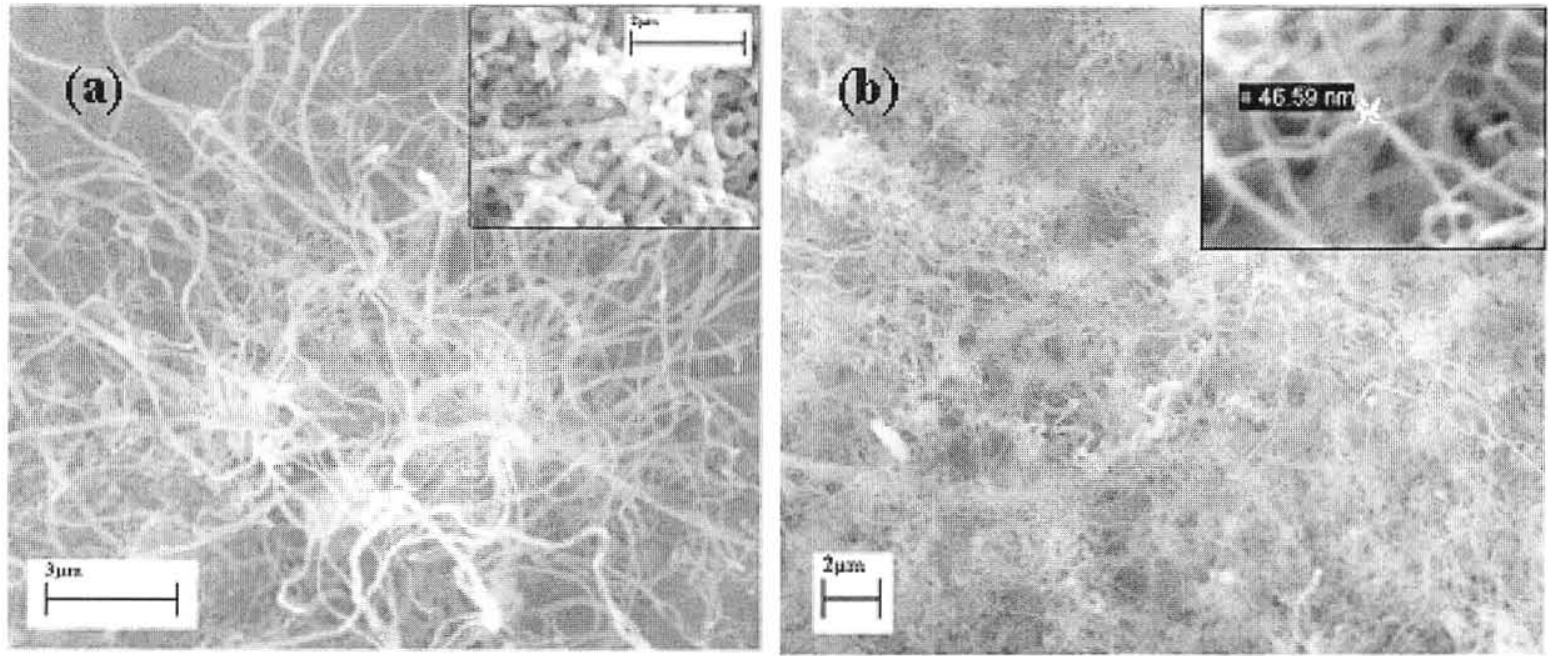

Fig.48 SEM images of Si NWs (a) with Au catalyst (b) with Fe catalyst 
The other method for silicon nanowire growth, form $\mathrm{Si}$ and iron target (explained in chapter II), was successful and SEM images show the growth product collected from the tube's wall has many nanowires. The average diameter of these nanowires was small and they were long. Gold catalyst particles observed at the tips of wires (Fig.49).

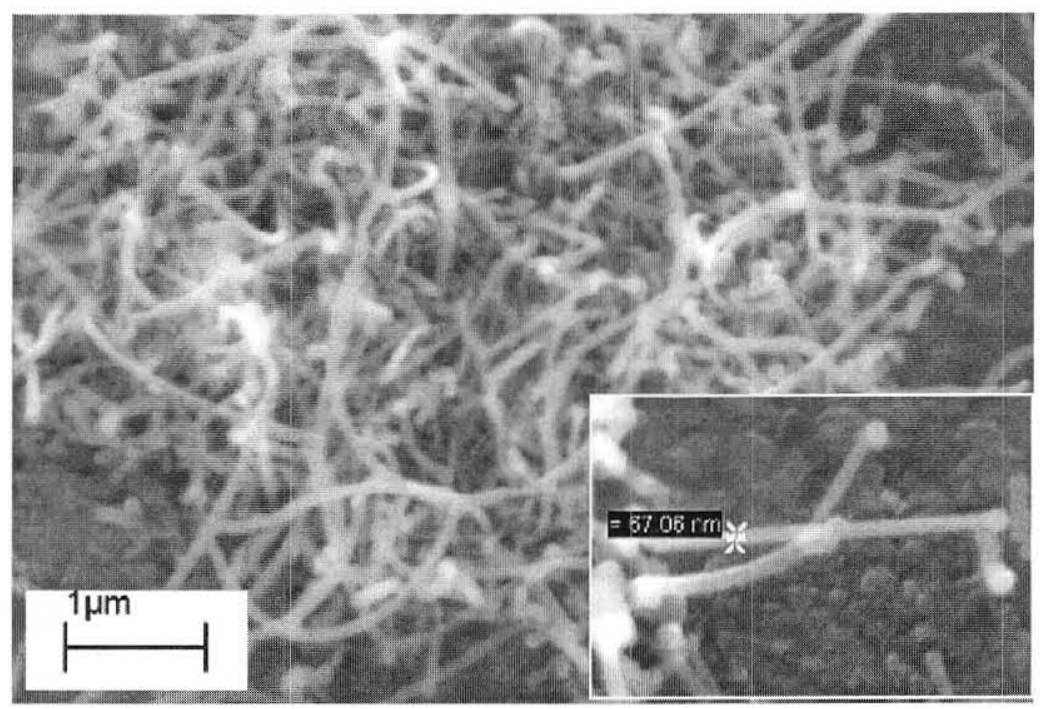

Fig.49 SEM

images of $\mathrm{Si}$

NWs with gold

catalyst

EDS showed the composition of nanowires was mainly $\mathrm{Si}$, oxygen and carbon was present as impurities. It is expected that the nanowires had an oxide layer on the surfaces since $\mathrm{Si}$ is very interactive with oxygen.

\section{Single wall carbon nanotubes}

The last part of this thesis is the result of PLV synthesis of SWNTs. After the synthesis process black colored materials covered the wall of the inner tube of the PLV system, and they could be scraped off easily. The material looked like some fluffy black soot.

One of the non-destructive techniques to prove the presence of SWNTs is Raman spectroscopy. Micro-Raman (Renishaw Raman microscope) was performed on several 
nanotubes, with a red laser, wavelength of $632.8 \mathrm{~nm}$ and power of $16-17 \mathrm{~mW}$. Two well separated peaks appearing $\sim 1555 \mathrm{~cm}^{-1}$ correspond to the tangential modes for metallic and semiconductor nanotubes. Low frequency modes $\sim 160 \mathrm{~cm}^{-1}$ correspond to radial breathing modes, which is radius dependent. Second order Raman peaks appear $\sim 2615$ $\mathrm{cm}^{-1}$. (Fig.50)

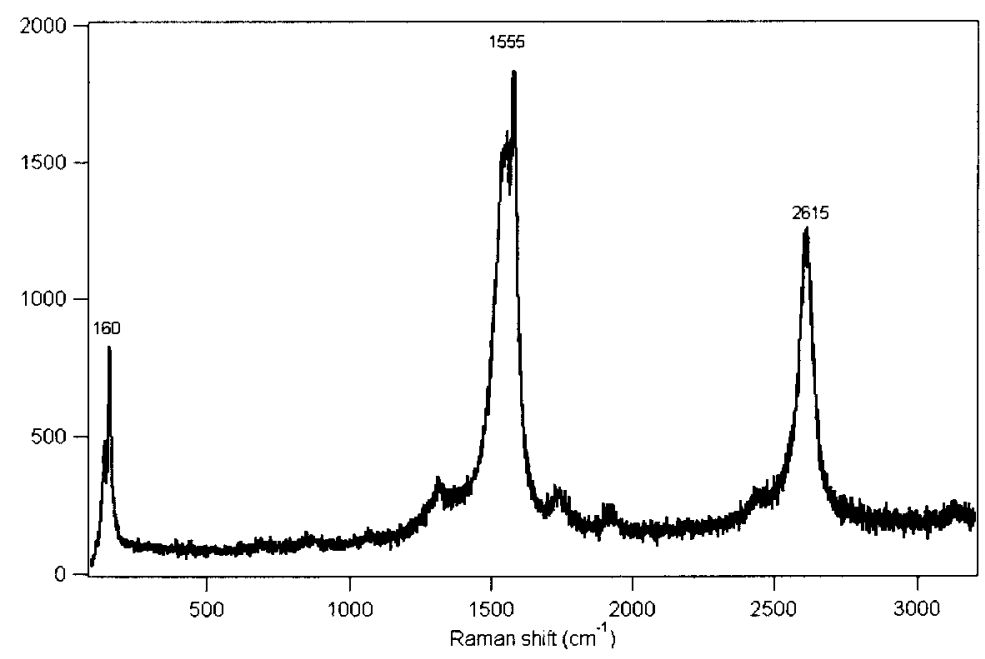

Fig.50

Raman spectrum of SWNT 


\section{CHAPTER IV}

\section{CONCLUSION}

$\mathrm{Ga}_{2} \mathrm{O}_{3}$ nanowires with herringbone and ribbon structures and $\mathrm{Ga}_{2} \mathrm{O}_{3}$ nanosheets were synthesized using three different methods. Te doped GaAs powder in argon inert atmosphere at $900{ }^{\circ} \mathrm{C}$ resulted in white color gallium oxide after physical vapor decomposition of GaAs and oxidation of $\mathrm{Ga}$ in the reactor. GaAs and Te mixed with gold powder produced gallium oxide nanostructures under similar experimental conditions. Also undoped $\mathrm{GaAs}$ mixed with $\mathrm{S}$ resulted in gallium oxide nanostructures. $\mathrm{Ga}_{2} \mathrm{O}_{3}$ nanostructures were characterized with SEM and TEM. The presence of Au reduces the diameter of the nanowires while $\mathrm{S}$ increases it. Some changes were observed in nanowires morphology for these methods as follow; $\mathrm{Te} / \mathrm{GaAs}$ results nanoribbons, adding some Au produced nanorods and $\mathrm{S}$ results in sheets. Herringbone structure observed for all methods and they are unique. AFM, EDS, XRD and Raman spectroscopy were done for more chemical and physical characterization of the synthesis products.

GaAs nanowires were synthesized through VLS growth in the PLV system using $\mathrm{Au}$ as catalyst. The diameter of the nanowires was small $\sim 30 \mathrm{~nm}$ in diameter and $\sim 10 \mu \mathrm{m}$ long. SEM and EDS were used to study the nanowires and provided more information.

$\mathrm{GaP}$ nanowires were grown in the PLV using $\mathrm{Au}$ as catalytic particles. Experiment showed, if the initial GaP crystalline pieces were ground for longer time, the diameter of the nanowires decreased and the length increased. SEM and EDS revealed more information about the appearance and constituent elements of the nanowires. 
$\mathrm{GaP} / \mathrm{GaAs}$ nanowires were synthesized by applying the pulse laser vaporization method. The structure of the nanowires was superlattice of crystalline Gap and GaAs. High resolution TEM is needed to observe the superlattice structure. Au and Fe used as catalytic particles dispersed on the Si substrate. Nanowires were grown on the substrates and the walls of the quartz tube. SEM, EDS, Raman spectroscopy were used for structure characterization. Some broadening and shifting were observed for $\mathrm{GaAs}$ and $\mathrm{GaP}$ peaks in the Raman spectroscopy result.

Si nanowires were grown using PLV, with $\mathrm{Au}$ and Fe catalysts. The catalysts used in two different ways; micropowder mixed with Si powder in the target, and catalyst nanoparticles were dispersed on the substrate.

SWNTs were synthesized by the vapor liquid solid (VLS) method in the PLV system. Raman spectroscopy showed peaks corresponding to tangential and radial breathing modes of metallic and semiconducting SWNTs. 


\section{REFERENCES}

[1] zhong lin Wang, CHARACTERIZATION OF NANOPHASE MATERIALS

[2] J. Chem. Phys., Vol. 119, No. 13, 1 October 2003

[3] J. Phys. Chem. B 2003, 107, 4527-4531

[4] P. Holister, J. W. Weener, C. Roman Vas, T. Harper; Cientifica

[5] Mildred Dresselhaus and Gene Dresselhaus, Peter Eklund, Riichiro Saito, Chofugaoka, Chofu-shi , Physics world, January 1998

[6] Appenzeller, J.; Martel, R.; Radosavlievic, M.; Heinze, S.; Avouris, P. Source: 2003 Third IEEE Conference on Nanotechnology. IEEE-NANO 2003. Proceedings (Cat. No.03TH8700), 2003, pt. 2, p 236-9 vol.2

[7] Jae-Yoo Kim, Moonhee Kim, Jong-Ho Choi. , Synthetic Metals 139 (2003) 565568

[8] R. Martel*, V. Derycke, J. Appenzeller, S. Wind, and Ph. Avouris, IBM T. J. Watson Research Center, Route 134, Yorktown Heights, NY 10598

[9] J. Appl. Phys. Vol. 42 (2003) pp. L 1288-L 1291 Part 2, No. 10B, 15 October 2003

[10] T. W. Ebbesen, Carbon Nanotubes preparation and property

[11] Z. L. Wang, Y. Liu, Z. Zhang, Handbook of Nanophase and Nanostructured Materials, V.1, V.3 
12] Z. L. Wang, Y. Liu, Z. Zhang ; Handbook of Nanophase and Nanostructured material, V 3

[13] R. Zhang, Y. Lifshitz, S. Lee; Adv. Mater. 2003, 15, No. 7-8, April 17

[14] C. M. Lieber; Adv. Mater. 2000, 12, No. 4

[15] Z. L. Wang, Y. Liu, Z. Zhang ; Handbook of Nanophase and Nanostructured material, V 1

[16] A. M. Morales, C. M. Lieber, Science. VOL. 279, 9Jan 1998

[17] X. D. Duan, C. M. Lieber, Advance materials, 2000, 12, No.4

[18] Y. H. Tang, Y. F. Zhang, H. Y. Peng, N. Wang, C. S. Lee and S. T. Lee, Chem. phys. Lett., V 314, Issue 1-2, 26 Nov.

[19] B. Fultz, J. M. Howe, Transmission Electron Microscopy and Diffractometry of Materials

[20] Q. Yang, J. Sha J. Xu , Y.J. Ji , X.Y. Ma, J.J. Niu , H.Q. Hua , D.R. Yang, Chemical Physics Letters 379 (2003) 87-90

[21] Patern in Nature, http://acept.la.asu.edu/PiN/rdg/elmicr/elmicr.shtml

[22] B. D. Cullity, Elements Of X-ray Diffraction

[23] Xiangfeng Duan, Jianfang Wang, and Charles M. Lieber, APPLIED PHYSICS LETTERS, VVOLUME 76, NUMBER 9

[24] G. Park, W. Choi, Y. C. Choi, Y. H .Lee, C.B. Lim, Journal of crystal Growth $220(2000) 494-500$

[25] H.W. Seo, S.Y.Bag, J.Park, H.Yang, S.Kim, Chem. Commun.,2002,2564-2565 [26] http://cimesgl.epfl.ch/CIOL/ems.html 


\section{CURRICULUM VITAE}

NAME:

Romaneh Jalilian

ADDRESS: $\quad$ Department of Physics

102 Natural Sciences Building

Louisville, KY 40292

D.O.B.:

May $2^{\text {nd, }} 1977$

EDUCATION:

B.C. Physics, University of Shahid behesti, Tehran, Iran,1995-2000

M.S. Physics

University of Louisville

AWARD: $\quad$ Manuel B. Schwartz Award for Outstanding Graduate Student (2004)

EXPERIMENTAL

SKILLS:

Experience in nanostructures synthesis applying PLV and CVD

Experience in material characterization applying SEM, XRD, EDS, XPS. 